\title{
Functionalized bead assay to measure 3-dimensional traction forces during $\mathrm{T}$-cell activation
}

\author{
Morteza Aramesh ${ }^{1}$, Simon Mergenthal ${ }^{2}$, Marcel Issler², Birgit Plochberger ${ }^{3}$, Florian Weber ${ }^{3}$, \\ Xiao-Hua Qin ${ }^{4}$, Robert Liska ${ }^{5}$, Georg N. Duda ${ }^{6}$, Johannes B. Huppa ${ }^{7}$, Jonas Ries ${ }^{8}$, Gerhard J. \\ Schütz ${ }^{9,{ }^{*}}$ and Enrico Klotzsch ${ }^{1,2, *}$
}

\footnotetext{
${ }^{1}$ Laboratory of Applied Mechanobiology, Department for Health Sciences and Technology, ETH Zürich, 8093 Zürich, Switzerland

${ }^{2}$ Institute of Biology, Experimental Biophysics/ Mechanobiology, Humboldt Universität zu Berlin, 10115 Berlin, Germany

${ }^{3}$ Upper Austria University of Applied Sciences, Campus Linz, Garnisonstrasse 21, 4020 Linz, Austria

${ }^{4}$ Institute for Biomechanics, Department for Health Sciences and Technology, ETH Zürich, 8093 Zürich, Switzerland

${ }^{5}$ Institute of Applied Synthetic Chemistry, TU Wien, Getreidemarkt 9/163/MC, 1060, Vienna, Austria

${ }^{6}$ Julius Wolff Institute for Biomechanics and Musculoskeletal Regeneration, Charité-Universitätsmedizin Berlin

7 Institute for Hygiene and Applied Immunology, Center for Pathophysiology, Infectiology and Immunology, Medical University of Vienna, Vienna, Austria

${ }^{8}$ EMBL Heidelberg, Meyerhofstraße 1, 69117 Heidelberg, Germany.

${ }^{9}$ Institute of Applied Physics, TU Wien, Vienna, Austria

* Correspondence should be send to:

schuetz@iap.tuwien.ac.at and enrico.klotzsch@hu-berlin.de
} 


\begin{abstract}
When T-cells probe their environment for antigens, the bond between the T-cell receptor (TCR) and the peptide-loaded major histocompatibility complex (MHC) is put under tension, thereby influencing the antigen discrimination process. Yet, the quantification of such forces in the context of T-cell signaling is technically challenging. Common approaches such as traction force microscopy (TFM) employ a global readout of the force fields, e.g. by measuring the displacements of hydrogel-embedded marker beads. Recent data, however, indicated that T-cells exert tensile forces locally via TCR-enriched microvilli while scanning the surface of antigen-presenting cells. Here, we developed a traction force microscopy platform, which allows for quantifying the pulls exerted via T-cell microvilli, in both tangential and normal directions, during T-cell activation. For this, we immobilized specific T-cell activating antibodies directly on the marker beads used to read out the hydrogel deformation. Microvilli targeted the functionalized beads, as confirmed by superresolution microscopy of the local actin organization. Moreover, we found that cellular components, such as actin, TCR and CD45 reorganize upon interaction with the beads, such that actin forms a vortex-like ring structure around the beads and TCR is enriched at the bead surface, whereas, CD45 is excluded from bead-microvilli contacts.
\end{abstract}

\title{
Significance statement (max 120 words)
}

During the antigen recognition process, T-cells explore and probe their environment via microvilli, which exert local pushes and pulls at the surface of the antigen presenting cell. It is currently believed that these forces influence or even enable the antigen recognition process. Here, we describe the development of a platform, which allows us to quantify the magnitude and direction of traction forces exerted locally by $\mathrm{T}$ cell microvilli. Simultaneous $\mathrm{Ca}^{2+}$ imaging was used to link the measured forces to the overall $\mathrm{T}$ cell activation status. Superresolution microscopy resolved the contact sites of bead-microvilli contact at the nanoscale: cells contacted beads via actin vortex-like structures, which excluded the phosphatase CD45 from the contacts. 


\section{Introduction}

T-cell receptors (TCRs) on the surface of T-cells recognize antigenic peptides on major histocompatibility complexes (pMHCs) of antigen presenting cells (APCs) with exquisite sensitivity and specificity (1-3). During the recognition process, the cellular interface - termed immunological synapse - undergoes substantial dynamic reorganizations, with T-cell microvilli contacting and searching the APC's surface for cognate antigen (4-6). It is still unclear, however, how the molecular binding events between pMHC and the TCR are transduced into intracellular signals (7). Mechanical force has recently been identified as an important parameter in the antigen recognition process $(8,9)$ : On one hand, tensile forces affect the interaction kinetics between TCR and PMHC (10-13), which appears to accentuate differences between agonistic and non-agonistic peptides $(14,15)$; On the other hand, there are indications that the TCR acts as a mechanosensor, which converts tensile forces into conformational changes, thereby affecting intracellular signaling $(16,17)$. In line with this, T-cells were shown to push and pull against model APCs (18-20). Vice versa, application of force via optically trapped pMHC-functionalized beads triggered T-cell activation, preferentially when forces were applied tangentially to the T-cell surface (21).

Different methods have been applied to quantify tensile forces directly in the synapse between a T-cell and an activating surface. DNA-based molecular tension sensors indicated the presence of pulling forces larger than $12 \mathrm{pN}$, when TCRs were engaged with antigenic glass-immobilized pMHCs $(21,22)$. Our own single molecule measurements using peptide-based force sensors revealed slightly lower forces around $5 \mathrm{pN}$ (23). When measured collectively via traction force microscopy, TCR-exerted sheer forces of up to $150 \mathrm{pN}$ were observed $(8,24)$. While these measurements allowed for quantification of tensile forces down to the level of individual bonds, they provide only indirect access to the directionality of these forces; particularly, forces acting perpendicularly to the T-cell surface have remained unknown. In addition, previous methods did not account for the highly localized force application by T-cells, which involves pulling on the APC surface via microvilli (4).

Here, we report the development of a traction force microscopy platform for the 3-dimensional quantification of tensile forces acting during T-cell activation. Compared to existing methods the platform shows two key advantages. Firstly, we generated well-defined nanoscopic points of force application via functionalized beads. The size of the beads of $200 \mathrm{~nm}$ diameter was chosen to match the width of microvilli (25). We used beads coated with anti-CD3 as specific 
TCR-ligand; in addition, we measured the influence of local co-stimulation via anti-CD28, which was expected to enhance signaling (9). Secondly, the beads were coupled to the surface of elastic gels, offering the possibility for 3-dimensional readout of their displacements. The platform allows for force measurements with a resolution of $56 \mathrm{pN}, 76 \mathrm{pN}$ and $93 \mathrm{pN}$ for tangential, normal and total forces, respectively. In addition, the T cell activation status was inferred from simultaneous $\mathrm{Ca}^{2+}$ measurements. Using this platform, we were able to directly quantify forces exerted via single T cell microvilli during the activation process. 


\section{Results and discussion}

\section{Functionalized bead assay for T-cell activation}

We selected a cytocompatible and photopolymerizable hydrogel system based on Polyethylene (glycol) diacrylate (PEGDA, $10 \mathrm{kDa}$ ), which has been widely used for regular cell culture and in situ cell photo-encapsulation studies (26). Compared to traditional polyacrylamide gels, PEGDA gels represent significantly lower cytotoxicity, comparable reactivity and minimal protein adsorption (27). The bead assay for activation of T-cells is shown schematically in Figure 1a. We designed it with respect to two main aspects:

i) The 3-dimensional displacements of the beads should be directly convertible into normal and tangential forces by solving the inverse stress-strain relationship (28). For this it is critical that individual beads can be treated as independent objects, which can be achieved by choosing sufficiently low bead densities so that their mutual distances are larger than the diameter of the contact area between bead and hydrogel surface $(29,30)$. In our experiments, the number of beads interacting with a single T-cell was in the range of 30 to 90 and the average attachment area was $175 \mu \mathrm{m}^{2}$, yielding average nearest neighbor distances of $0.7-1.2 \mu \mathrm{m}$ (31). For the chosen bead diameter of $200 \mathrm{~nm}$ beads can hence be treated as independent objects for traction force characterizations (32). We used neutravidin-conjugated beads, which were bound to the surface of photo-crosslinked, biotinylated poly(ethylene glycol) diacrylate (PEGDA) gels.

ii) Forces exerted by T-cells onto the surface should act strictly locally via the functionalized beads. We coupled biotinylated anti-CD3 and/or anti-CD28 to the beads to trigger the TCR's $\varepsilon$-subunit alone or in combination with the co-stimulatory protein CD28. By choosing beads of $200 \mathrm{~nm}$ diameter we aimed at matching the size of naturally occurring microvilli, with which T-cells contact the surface of APCs during the search for antigen $(5,6,33)$. Using neutravidin-coated beads makes the assay flexible, as beads could be functionalized also with different biotinylated antibodies or peptides to stimulate the target cells.

In this paper we showcase the performance of our platform using Jurkat $T$ cells as a model system; early T-cell activation was assessed via the $\mathrm{Ca}^{2+}$-sensitive ratiometric dye Fura-2 (34). We first validated the functionality of the platform by quantifying T-cell activation efficiency on gels of different stiffness (Figure 1b). Consistent with previous literature (35), we observed a pronounced increase in the fraction of activated cells with increasing stiffness of the PEGDA gel; eventually, beads immobilized directly on poly-d-lysine-coated rigid glass surfaces yielded maximum activation efficiency (data points at $20 \mathrm{GPa}$ stiffness for glass (36)). The delay 
between surface contact and T cell activation was longer for soft gels compared to stiff surfaces

(Figure 1c), with the presence of CD28 further reducing this delay. Also this finding was expected, as it is known that stronger stimuli yield faster $\mathrm{Ca}^{2+}$ responses (37).

a)

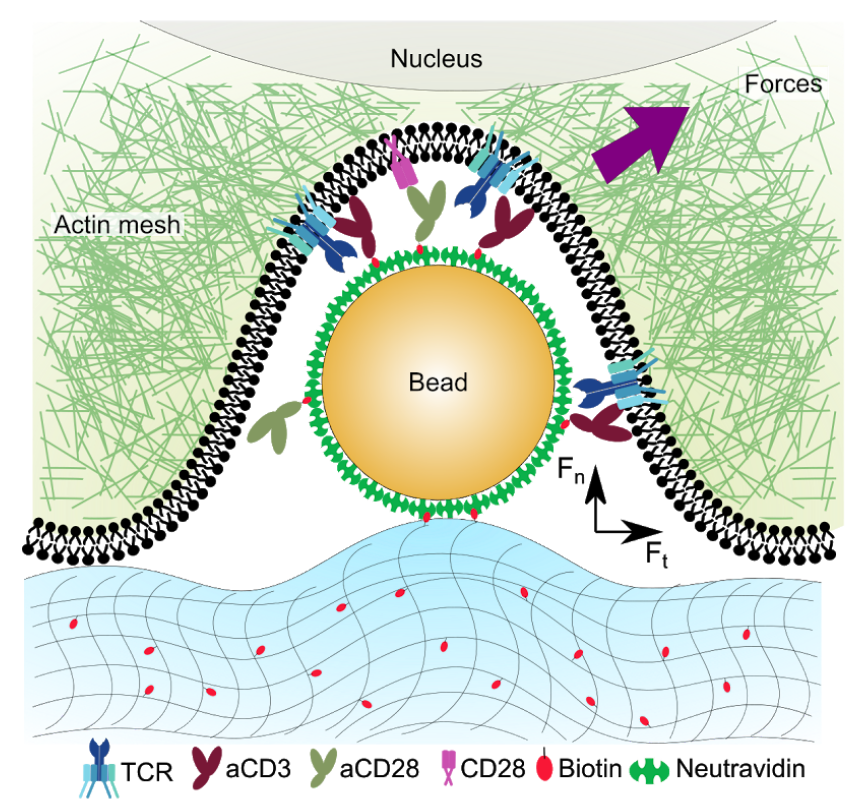

b) Activation dependence on hydrogel stiffness

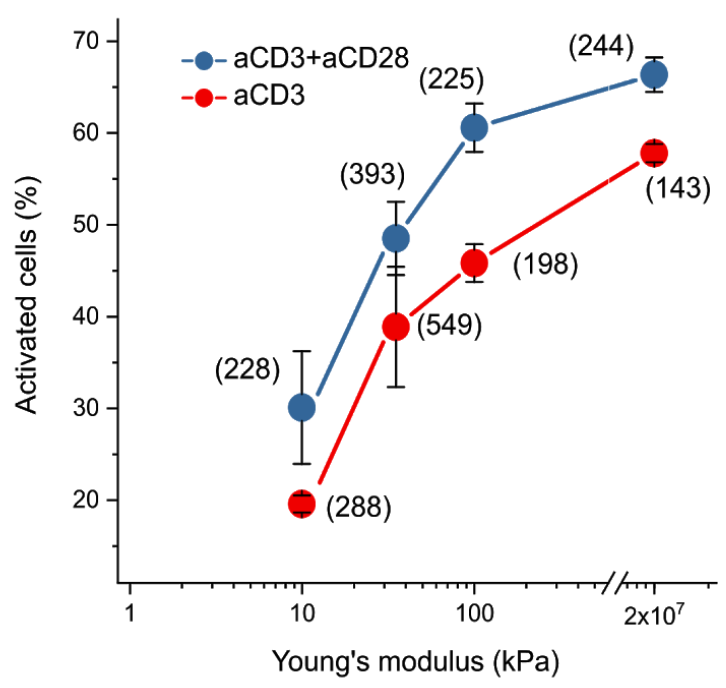

c)

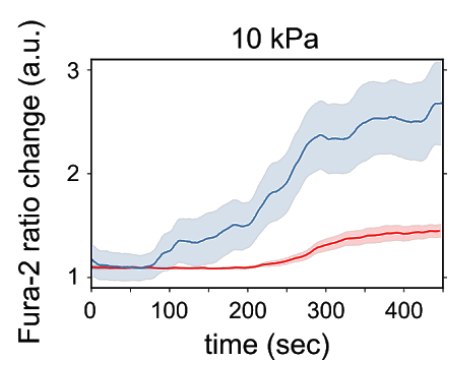

Calcium signaling during cell activation

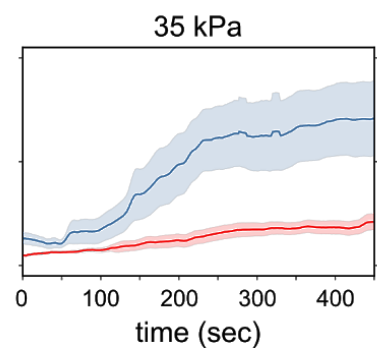

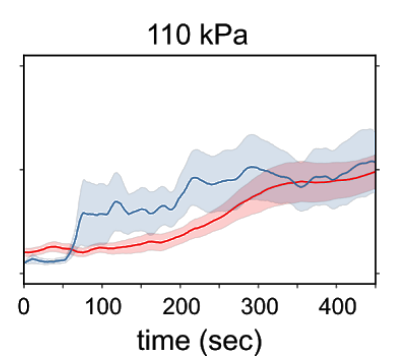

$-\mathrm{aCD} 3+\mathrm{aCD} 28$ $-\mathrm{aCD} 3$

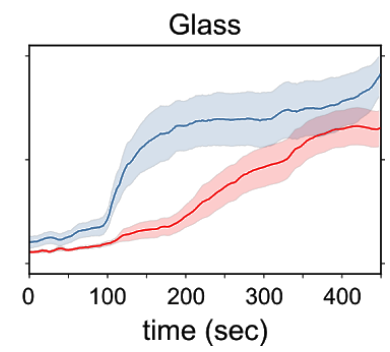

Figure 1: Setup for T-cell activation and $\mathrm{Ca}^{2+}$ imaging. (a) Schematic of the traction force microscopy assay. Fluorescent neutravidin-coated beads with a diameter of $200 \mathrm{~nm}$ were coupled to UV-crosslinked biotinylated PEGDA hydrogels. Beads were functionalized with anti-CD3 and anti-CD28 prior to seeding the cells. (b) Effect of hydrogel stiffness on T cell activation. The percentage of activated T cells, defined by a minimal Fura-2 ratio of 1.5 , was recorded for PEGDA hydrogels of different stiffness. Data obtained at $20 \mathrm{GPa}$ stiffness correspond to experiments, in which beads were coupled directly to the glass surface. Red and blue data points correspond to experiments performed with beads coated with anti-CD3 only, or with a mixture of anti-CD3 and anti-CD28 antibodies. The number of analyzed cells is given in brackets. (c) Kinetics of $\mathrm{Ca}^{2+}$ signaling. Fold change of Fura-2 ratio is plotted over time for the different PEGDA hydrogel stiffnesses used in (b), for anti-CD3 with (blue) or without (red) anti-CD28. Fura-2 ratio at the time of the cell first touching down till the onset of $\mathrm{Ca}^{2+}$ release (slope increasing) was averaged to calculate Fura- 2 ratio change over time. Solid line shows the average over a minimum of 10 cells, shaded areas the standard error of the mean (s.e.m.). 


\section{Combined 3-dimensional Traction Force Microscopy and Calcium imaging}

To investigate more closely the generation of 3-dimensional forces in the context of early T-cell signaling, we used substrates of medium stiffness (35 kPa). Beads were imaged via fluorescence microscopy, and their displacements were determined with a precision of $5 \mathrm{~nm}$ in $x y$ and $30 \mathrm{~nm}$ in z-direction. Bead displacements were translated into forces as described in the Methods section. Without cells, the determined bead positions showed only small variations, corresponding to force fluctuations of $56 \mathrm{pN}, 76 \mathrm{pN}$ and $93 \mathrm{pN}$ for tangential, normal and total forces, respectively (Figure $\mathbf{2 d}$, control). We used these residual fluctuations to define the force resolution of the platform.

In a typical experiment, T-cells were seeded onto the platform, and we measured both 3D bead displacements and $\mathrm{Ca}^{2+}$ traces simultaneously over several minutes (Figure 2). Exemplary time traces of the force evolution per bead during the process of T-cell activation are shown in Figure 2c; single beads experienced forces up to 500 pN. For a global analysis, we determined the forces acting on individual beads at the time-point of T-cell activation, which we defined by the maximal $\mathrm{Ca}^{2+}$ flux for the cell analyzed (Figure 2d). Interestingly, tangential forces showed no correlation with normal forces, indicating that the two directions are largely uncoupled (Figure S1). T-cells exerted substantially higher forces to anti-CD3/anti-CD28 co-functionalized beads than to beads functionalized only with anti-CD3 (Figure 2d), in line with the observation of Bashour et al. (8).

Finally, since T-cells can exert tensile forces exclusively via the beads, our assay allows us to calculate the accumulated force exerted by single T-cells. Exemplary data are shown in Figure S2, where we plotted the accumulated force versus the $\mathrm{Ca}^{2+}$ response as parametric representation. Not surprisingly, increased force generation correlated with increased $\mathrm{Ca}^{2+}$; when $\mathrm{Ca}^{2+}$ levels declined at later stages, also forces were reduced again. In total, $\mathrm{T}$ cells exerted up $10 \mathrm{nN}$ of force onto the stimulating beads. Given a pulling force of $5-10 \mathrm{pN}$ exerted by single TCR molecules $(22,23)$, we estimate between 1000 and 2000 TCR molecules involved in the activation process. 
a)

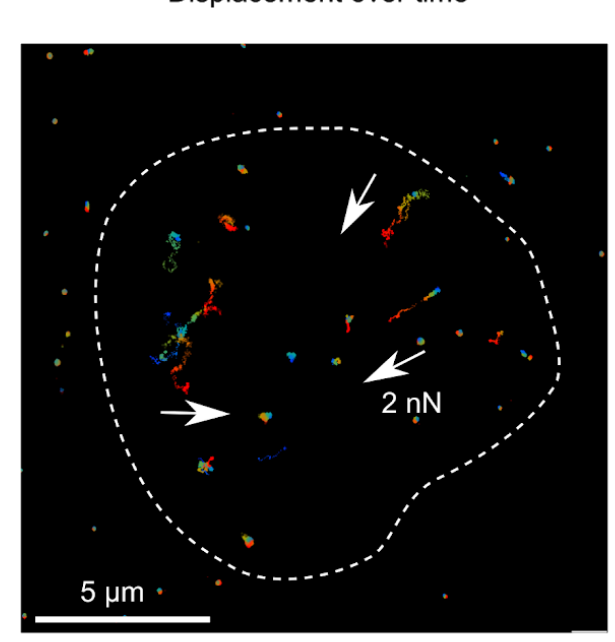

b)

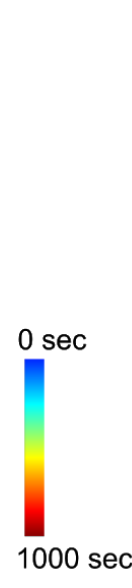

Displacement in normal direction

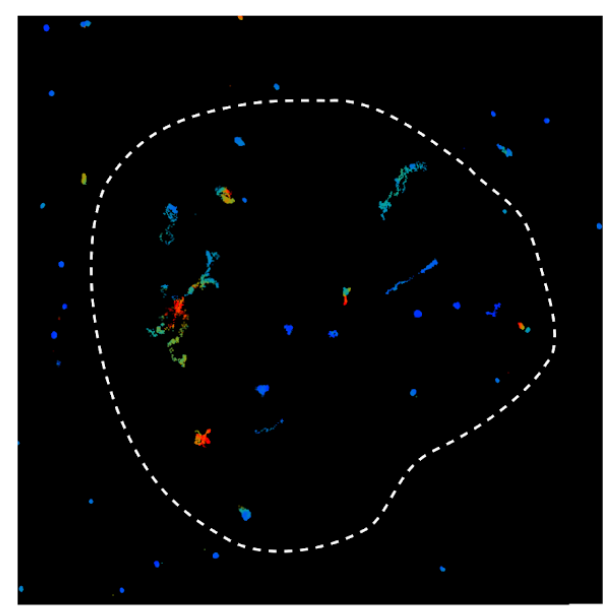

$0 \mathrm{~nm}$

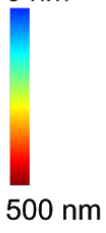

c) forces per bead for single cell

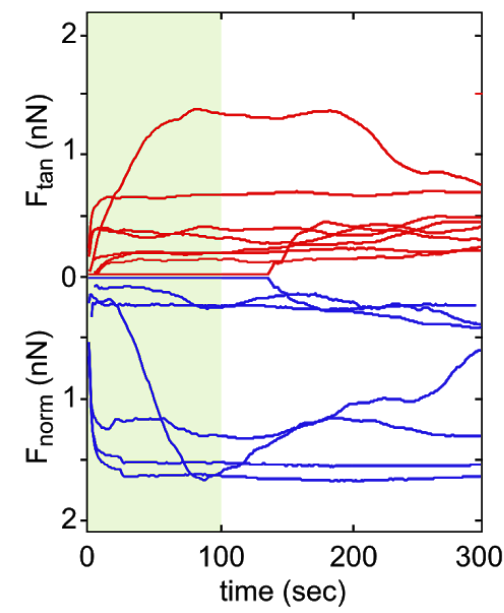

d)

Figure 2: Traction force measurements (a) Bead displacements are plotted over time for an exemplary cell. We used a PEGDA hydrogel with a Young's modulus of $35 \mathrm{kPa}$ and beads coated both with anti-CD3 and anti-CD28. The left panel shows tangential bead displacements, with color-code indicating time. The white dashed line shown in $(\mathbf{a}, \mathbf{b})$ indicates the cell outline, derived from the Fura-2 signal. The white arrows represent a bead displacement equivalent to $2 \mathrm{nN}$. (b) Same cell as shown in (a), with color code indicating the z-displacements of the beads. (c) Normal (blue) and tangential (red) forces experienced by beads underneath the cell are shown within the first $300 \mathrm{~s}$ upon cell attachment. Data correspond to the same cell as in panel $(\mathbf{a}, \mathbf{b})$. Green shading indicates the time prior to $\mathrm{Ca}^{2+}$ flux, defined by a 1.5 -fold increase in the Fura-2 ratio. (d) The Violin/Whisker-Box plots summarize all recorded forces at the time point of $\mathrm{Ca}^{2+}$ flux, with blue, red and purple indicating normal, tangential, and total forces. As control, we determined the maximal force, beads without cell contact were exposed to, representing thermal fluctuations and precision of the bead assay. We defined here as force resolution the mean plus standard deviation, yielding $56 \mathrm{pN}, 73 \mathrm{pN}$ and $93 \mathrm{pN}$ for tangential (red), normal (blue) and total (violet) force, respectively. Data represent at least ten cells and 160 beads per condition. 


\section{Nanoscale actin ring formation at T-cell-bead contacts}

We next aimed at studying the mechanisms of force generation during T-cell activation in more detail. For this, we imaged filamentous actin via expression of tractin-cyOFP (38) (Figure 3). We employed structured illumination microscopy (SIM) to visualize the actin distribution at the cells' basal membrane next to the beads at sufficiently high resolution. In order to perform SIM, however, the PEGDA hydrogel had to be replaced by a material with high refractive index (39); we selected the PDMS-based QGel920 featuring a stiffness of $18 \mathrm{kPa}$, similar to PEGDA of medium stiffness.

SIM images indicate the formation of actin rings around the anti-CD3/anti-CD28 functionalized beads (Figure 3a). For a global analysis of all structures, we segmented the actin images into regions around individual beads and averaged over all beads within the cells' attachment area (Figure 3c). Directly after cell attachment an actin ring is formed around the beads (Figure 3b, left), reminiscent of actin vortices described previously for cortical actin (40). Closer inspection of the ring morphogenesis, however, revealed surprising details: typically, single microvilli contacted a single bead tangentially, which - within a few tens of seconds - initiated the engulfment of the bead (Figure 3b). The distance from the beads' center to the actin ring increased over time, and eventually the actin ring completely disappeared at late stages (Figure 3c, Movie S1).

To quantify the images, we plotted the radial distribution over time (Figure 3d and Figure S3). To avoid artifacts arising from overlapping signals, beads with a mutual distance smaller than $200 \mathrm{~nm}$ were excluded from the analysis. Immediately after T-cell spreading the actin ring had a radius of $\sim 275 \mathrm{~nm}$ and a width of $\sim 250 \mathrm{~nm}$ (blue curve), further supporting the interpretation that single microvilli were wrapping around individual beads. During this process, the interaction between anti-CD3 on the bead surface and the TCR the microvillus surface stabilizes a strongly bent conformation of the actin bundle in the microvillus core. Given the flexural stiffness of actin rods of $\sim 50 \mathrm{pN} / \mathrm{nm}$ (41), single microvilli can easily account for the observed tangential forces of a few $100 \mathrm{pN}$ acting on single beads. At later stages, we observed a shift to $500 \mathrm{~nm}$ radius and a much broader and less dense actin distribution (red curve), indicating a massive rearrangement of the membrane morphology close to the bead. Together, the morphogenesis, size, and the stabilization by actin (5) confirm the formation of microvilli-like protrusions, which were contacting single beads. 
a)

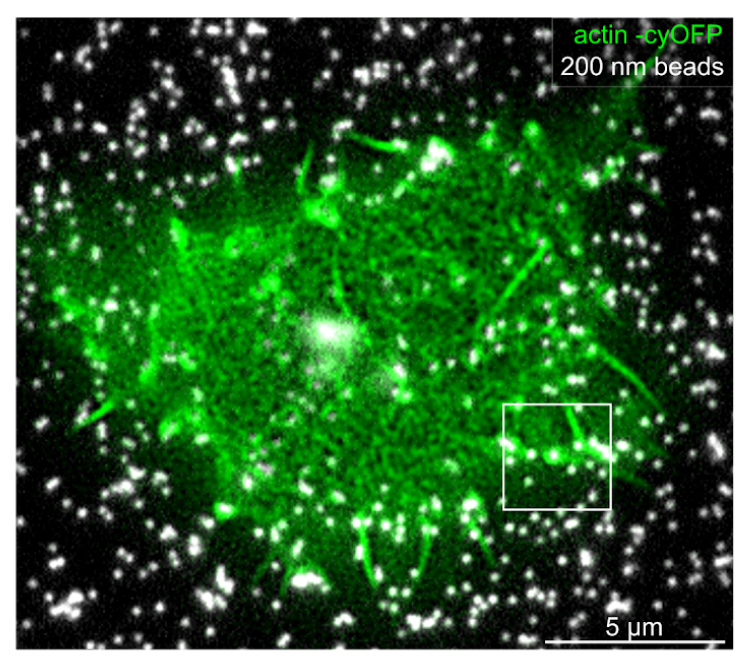

c)
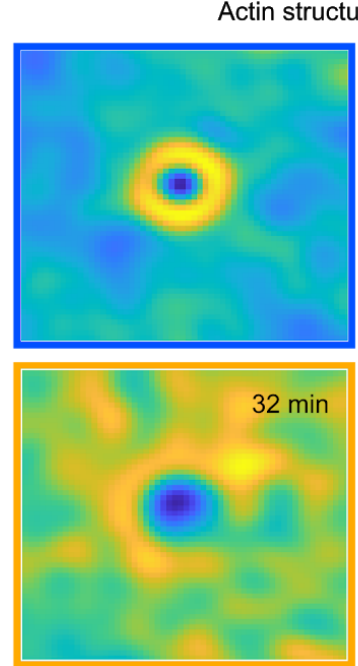

$16 \min$ b)
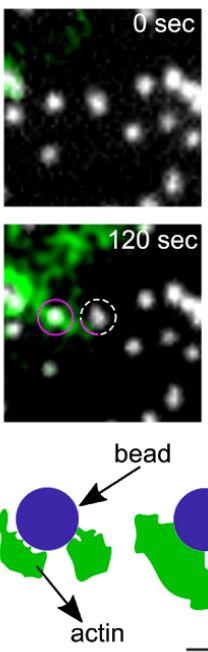

Microvilli - bead interaction
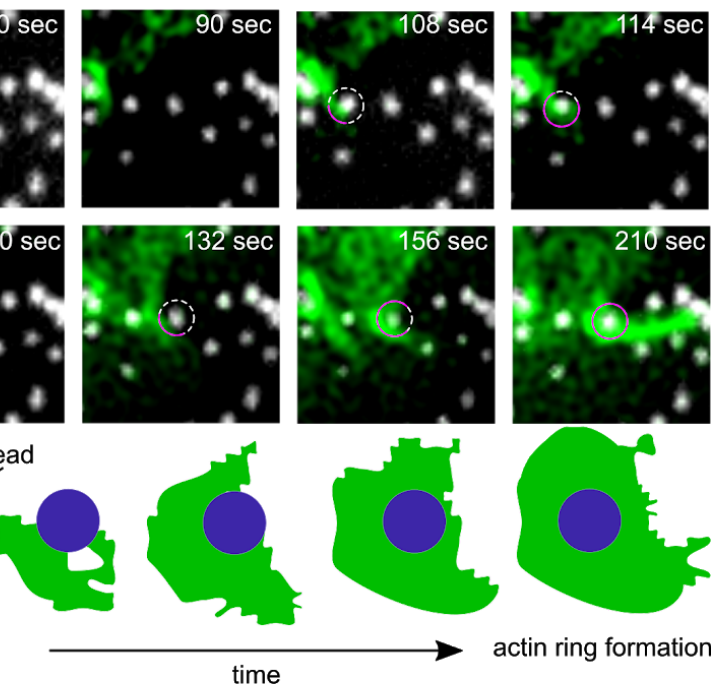

time

d) Time-dependent spreading of actin around a bead

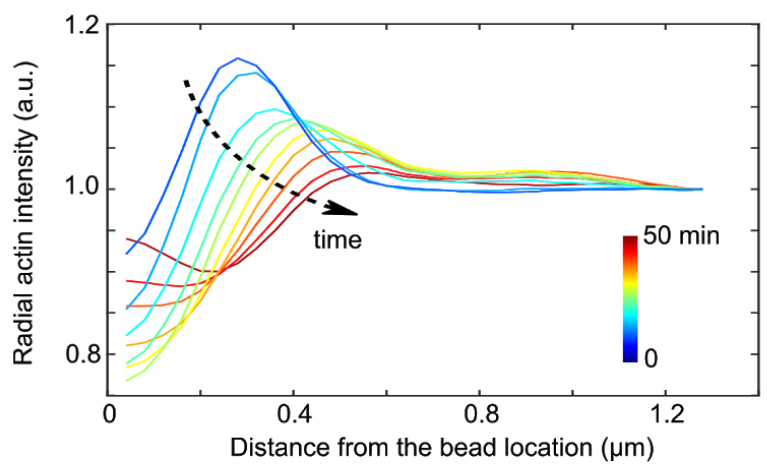

Figure 3: Actin ring formation. (a) SIM image of cellular actin (green) overlaid with the calculated bead positions (white). The experiment was performed with anti-CD3/anti-CD28 coated beads. (b) The time course of contact formation between a single microvillus and a single bead is exemplarily shown for the detail indicated by the white box in (a). At time point 108s the microvillus-bead contact is established. Within 6s, the microvillus has wrapped around the bead. Over the next two minutes an additional bead was contacted by a microvillus. The schematics below depict the temporal evolution of actin enrichment around the second bead that was contacted by the microvillus. (c) The averaged actin intensity around the beads is shown over time. The $n=76$ beads were included in the analysis. (c) The radial actin intensity with respect to bead positions is plotted as time evolution. Additional data of 10 cells fixed and stained with phalloidin A647 after $15 \mathrm{~min}, 30 \mathrm{~min}$ and $60 \mathrm{~min}$ with similar behavior are shown in Figure S3. The movie representing the data can be found in Movie S1. 


\section{TCR bead enrichment and CD45 exclusion on bead-microvilli contacts}

Finally, we were interested whether molecular rearrangements of signaling proteins accompany the observed formation of microvilli-bead contacts. Particularly, we were interested in the distribution of the large phosphatase CD45 relative to the bead surface: previously it was hypothesized that size-mediated CD45 exclusion from TCR-ligand contacts shifts the ITAM phosphorylation-dephosphorylation balance, thereby triggering TCR signaling (6).

Cells were fixed at different times of activation and labeled with respective antibodies for TCR and CD45 (Figure 4a). We applied Airyscan microscopy (42) in order to resolve the molecular organization of the TCR and CD45 as well as the positions of anti-CD3/anti-CD28 coated beads.

For quantitative analysis, we segmented the images in Figure $\mathbf{4 a}$ and determined the protein distribution with respect to the beads' positions. To avoid artifacts arising from overlapping signals, beads with a mutual distance smaller than $200 \mathrm{~nm}$ were excluded from the analysis. In Figure 4b we show the TCR- and CD45-distribution obtained from at least 200 registered beads. As expected, we observed the TCR to be associated with the bead surface, irrespective of the time point of fixation. In contrast, CD45 was efficiently excluded from the beads; the effect became more pronounced at later stages of $\mathrm{T}$ cell activation. 
a) Airy scan microscope images
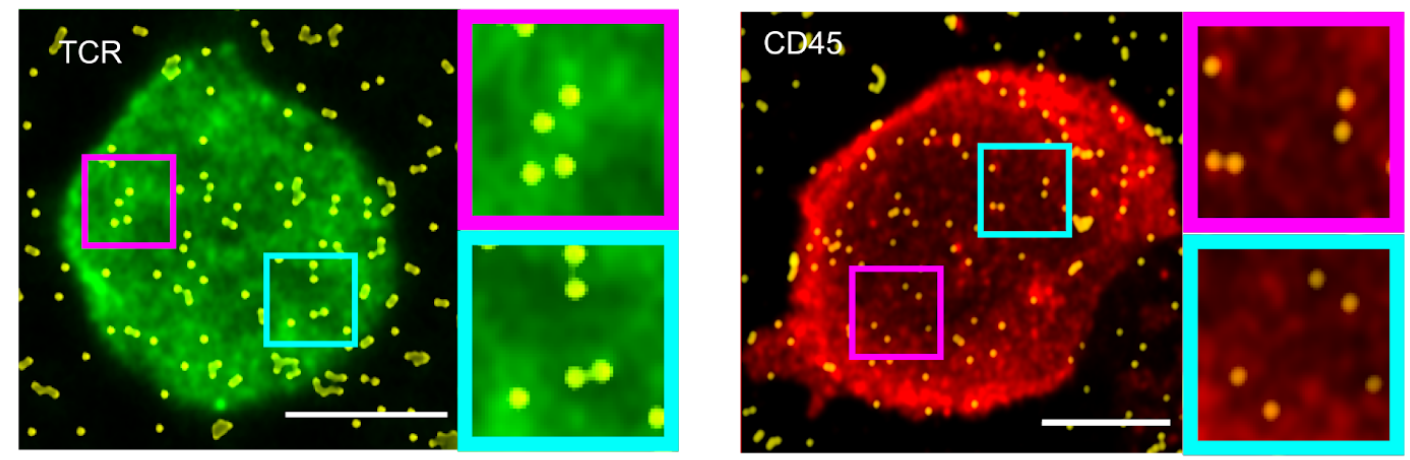

b)

Average profile of TCR and CD45 around the beads
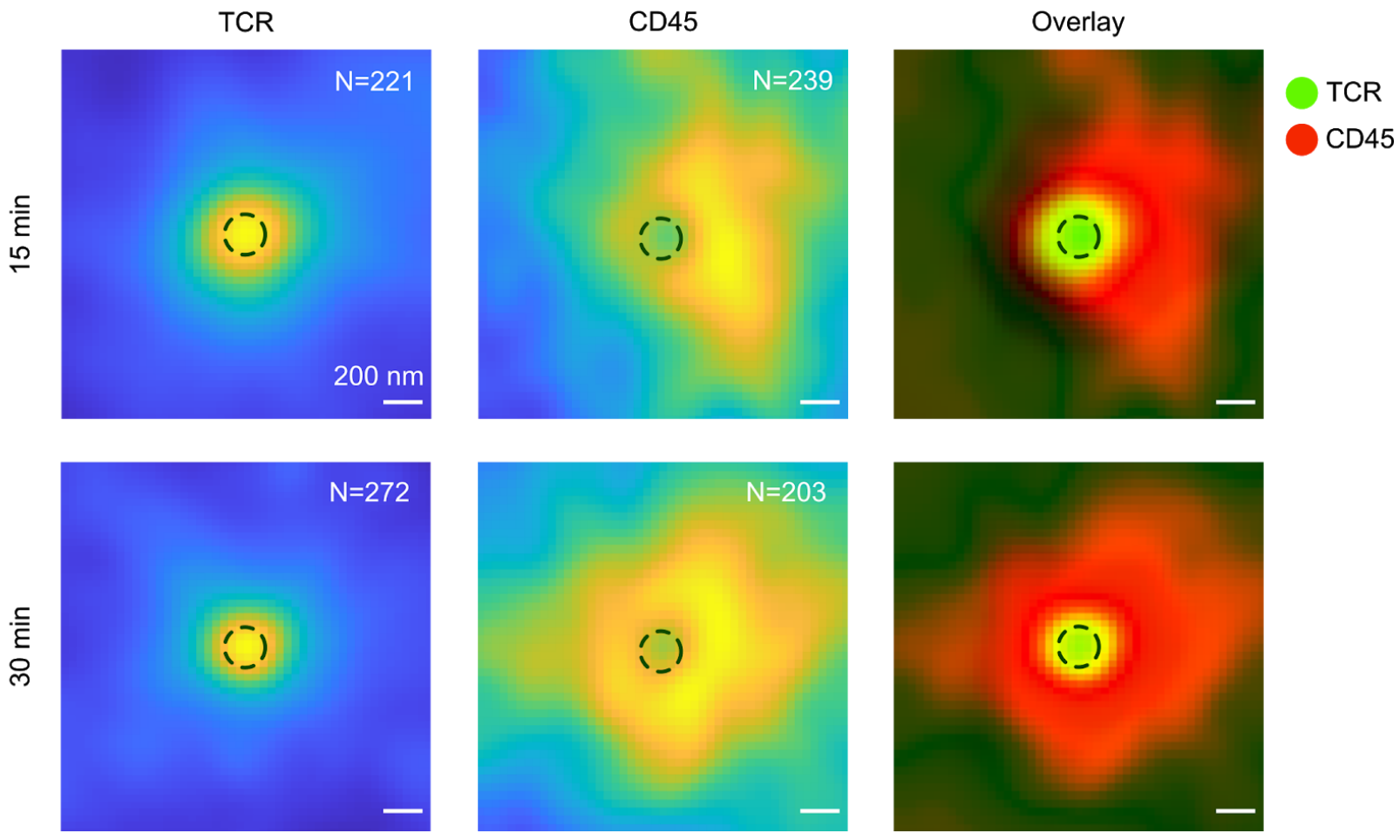

Figure 4. Molecular organization of TCR and CD45 with respect to the beads. Data were obtained using Airy scan microscopy on samples fixed at $15 \mathrm{~min}$ or $30 \mathrm{~min}$ after T-cell seeding. Experiments were performed using anti-CD3/anti-CD28 loaded beads. In (a) beads in yellow are overlaid with the TCR (left, green) and CD45 (right, red) signal; the image corresponds to fixation 15 min post seeding. Two magnified regions are shown on the right side. Scale bar is $5 \mu \mathrm{m}$. (b) Average intensity profile of registered beads for the TCR- (left) and the CD45-channel (middle) for 15 min (top row) and $30 \mathrm{~min}$ (bottom row) ( $\mathrm{N}$ indicates the number of analyzed beads, with at least 5 cells analyzed). Right column shows the overlay. Scale bar is $200 \mathrm{~nm}$. Dotted line is shown as a guide for the eye to indicate the region of the activating bead. 


\section{Conclusion}

In summary, by combining classic with pointillistic TFM, we present a versatile assay to measure and analyze cellular forces in the context of T-cell activation, offering a high potential for functional imaging by modification of bead functionalization. We showed that $\mathrm{Ca}^{2+}$ release as one early T-cell activation marker correlates with the mechanical stiffness of the underlying substrate, suggesting the suitability of the method for studying interfaces of cells with novel materials. By implementing fluorescence microscopy, we established 3D traction force microscopy with a $5 \mathrm{~nm}$ lateral and $30 \mathrm{~nm}$ vertical resolution, enabling high resolution mapping of tangential, normal and total force down to $56 \mathrm{pN}, 76 \mathrm{pN}$, and $93 \mathrm{pN}$, respectively. Superresolution microscopy revealed initial microvilli-bead contacts, which transformed within seconds to more complex engulfment of the beads, including the formation of vortex-like actin structures. Since the biotinylated ligands can be easily swapped, this methodology and bead assay is applicable also to the study of different cellular signaling mechanisms involving surface-anchored ligands, as in cell adhesion or intercellular contacts. 


\section{Materials and Methods}

Surface Modification and Preparation. Glass coverslips (CS-18R15, Warner Instruments) were either surface functionalized using plasma cleaning for activation followed by vapor phase silanization with Dichlorodimethylsilane (Sigma-Aldrich), resulting in a hydrophobic surface for sandwiching the curing hydrogel or with 3-(Trimethoxysilyl)propylmethacrylate (Sigma Aldrich) resulting in free methacrylate groups for crosslinking of PEGDA hydrogels to the surface.

PEG. Polyethyleneglycol (PEG, $M_{n} \sim 10 \mathrm{kDa}$ ) was purchased from Sigma-Aldrich (Austria). A water-soluble photoinitiator, Irgacure 2959 (12959) purchased from BASF (Germany). A heterofunctional PEG-based linker (Biotin-PEG5k-Thiol, Biotin-SH) was purchased from Nanocs Inc (USA). All other reagents were purchased from Sigma-Aldrich and used as received unless otherwise noted.

PEG Acrylation. PEG diacrylates (PEGDA, $10 \mathrm{kDa}$ ) were synthesized according to a generic protocol described by Hubbell et al. (43). Briefly, PEG $(10 \mathrm{~g}, 2 \mathrm{mmol}-\mathrm{OH})$ was dried by azeotropic distillation in $150 \mathrm{~mL}$ of toluene for $2 \mathrm{~h}$. After cooling to $40{ }^{\circ} \mathrm{C}$ under argon, triethylamine (1.4 mL, 5 equivalents) was added. Afterwards, acryloyl chloride $(0.7 \mathrm{~mL}, 4.5$ eq.) was added drop wise. The reaction was stirred overnight at room temperature under argon. The resulting solution was filtered over the alumina bed. The filtrate was subsequently neutralized with sodium bicarbonate and then concentrated by rotary evaporation. The crude product was re-dissolved in $20 \mathrm{~mL}$ of dichloromethane and precipitated in cold diethyl ether. After drying in vacuum, PEGDA was obtained as white solid at $\sim 85 \%$ yield. Acrylation degree of PEGDA was $\mathrm{x}$ approx. $95 \%$, as confirmed by ${ }^{1} \mathrm{H}-\mathrm{NMR}$ analysis (SI, Figure S4). ${ }^{1} \mathrm{H}-\mathrm{NMR}$ (CDCl3): $\delta(\mathrm{ppm})$ : $6.40\left(2 \mathrm{H}, \mathrm{m}, \mathrm{CH}_{2}=\mathrm{CH}-\right), 6.20\left(2 \mathrm{H}, \mathrm{m}, \mathrm{CH}_{2}=\mathrm{CH}\right.$ trans $), 5.80\left(2 \mathrm{H}, \mathrm{m}, \mathrm{CH}_{2}=\mathrm{CH}\right.$ cis $), 3.80-3.30(280$ $\times 4 \mathrm{H}, \mathrm{s},-\mathrm{CH}_{2}-\mathrm{CH}_{2}-$ ). It has to be noted that the efficiency of radical-mediated thiol-acrylate conjugation is as high as $95 \%$, (44) which was known to be higher than that of thiol-acrylamide conjugation (45).

Photo-Rheology. A plate-to-plate time-resolved photo-rheometer (Anton-Paar MCR-301, SI, Figure S5) was used to monitor the light-induced gel formation and quantify the bulk mechanical properties of PEGDA hydrogels. Filtered UV-VIS light $(320-500 \mathrm{~nm}$, Omnicure S2000) was directed from the bottom of the rheometer through a glass plate to the sample. Light intensity at the cure place was $10 \mathrm{~mW} \mathrm{~cm}^{-2}$ as determined by an Ocean Optics USB $2000+$ spectrometer. After a $60 \mathrm{~s}$ blank period, light was triggered to irradiate the samples. Real-time measurements were made in oscillation mode, at $25^{\circ} \mathrm{C}, 10 \%$ strain, $10 \mathrm{~Hz}$ and $20 \mu \mathrm{m}$ gap thickness. Strain and frequency sweeps were performed before and post the polymerization to verify the linear response regime. Plateau value of the storage modulus ( $\left.G^{\prime}\right)$ of the photopolymerized gels was termed as the bulk gel stiffness.

Hydrogel Preparation. Briefly, biotin-presenting PEGDA hydrogel layers with a thickness of 20 $\mu \mathrm{m}$ were prepared using a multistep procedure. First, PEGDA and Biotin-SH were dissolved in PBS solution of $0.5 \%$ Irgacure (I2959), achieving a final concentration of $30 \%$ and $0.16 \%$, 
respectively. The resultant solution corresponds to PEGDA gels with elastic moduli of $\sim 100 \mathrm{kPa}$. In order to access lower stiffness, the $30 \%$ precursor solution was further diluted with 0.5 I2959/PBS solution to a lower PEGDA content of $15 \%$ (for $35 \mathrm{kPa}$ ) and $5 \%$ (for $10 \mathrm{kPa}$ ), respectively. Second, $30 \mu \mathrm{L}$ of the precursor solutions were transferred to the central area of a cover slip (diameter: $30 \mathrm{~mm}$ ), which was pre-functionalized with methacrylate groups for covalent fixation (see above). The obtained specimen was then mounted on the top of the glass plate of a photo-rheometer setting (SI, Figure S6). The rheometer platform was lifted down to keep the sample thickness of $20 \mu \mathrm{m}$. Finally, the samples were exposed to UV irradiation for $300 \mathrm{~s}$ while at the same time performing photo-rheology measurements in order to measure bulk stiffness of the hydrogel.

For SIM and AiryScan microscopy we used QGels920 components A and B (CHT, Quantum silicones) with refractive index of 1.51 , in a ratio of $1: 5$ arriving at a stiffness of $18 \mathrm{kPa}$ using AFM tip indentation (Figure S7) (39). The surface was further modified using UV irradiation for 3 min, Sulfo-NHS-LC-biotin (ThermoFisher) dissolved to $1 \mathrm{mg} / \mathrm{mL}$ in a Phosphate buffer at $\mathrm{pH} 8.6$ and $50{ }^{\circ} \mathrm{C}$ Celsius. The biotinylated surface was then incubated with FluoSpheres ${ }^{\mathrm{TM}}$ NeutrAvidin ${ }^{\mathrm{TM}}$-marked microspheres $(200 \mathrm{~nm}$ in diameter, fluorescent in yellow-green, ThermoFisher, F8774) with 1-5uL in 150uL PBS per hydrogel. Biotinylated CD3 (OKT3, ThermoFisher) and CD28 (CD28.2, BioLegend) specific antibodies were added concomitantly at $5 \mathrm{ug} / \mathrm{mL}$, RT and for at least 30 minutes.

\section{PEGDA hydrogel characterization for TFM measurements.}

Bulk stiffness measurements accessed by photo-rheometry allow classification of cells' ensemble behavior, we were interested in the sensation of the local stiffness as the cell senses force on the individual bead level(46). For this, AFM tip indentation (see paragraph below) was used to probe the PEGDA surface stiffness locally and also to test for lateral homogeneity of the hydrogels (Figure S8, S9). For that, BSA-coated AFM tips were scanned over five regions of 90 $\times 90 \mu \mathrm{m}^{2}$ with $16 \times 16$ points of indentation, revealing an average Young's modulus of $14 \pm 5$ $\mathrm{kPa}, 33 \pm 14 \mathrm{kPa}$ and $95 \pm 19 \mathrm{kPa}$ for the soft, medium and hard hydrogel stiffness maps using a tip velocity of $2 \mu \mathrm{m} / \mathrm{s}$ with a maximal indenting force of 30-400 pN (see Figure S8). Furthermore, in order to test for gel elasticity, we checked for the independence of Young's modulus from the loading rate of indentations at the same position (see Figure S9), with a slight trend towards material softening at higher speeds between 25 and $500 \mathrm{pN} / \mathrm{s}$. This allowed us to exclude non-elastic stress relaxation contributions to T-cell behavior as was measured to be important for cell size regulation by Mooney et al.(47).

AFM stiffness measurements. PEGDA and PDMS hydrogels were characterized with Atomic Force Microscopy (NanoWizard 3, JPK, Berlin). Soft silicon nitride AFM probes with nominal spring constants of $\mathrm{k} \sim 0.01 \mathrm{~N} / \mathrm{m}$ and $0.02 \mathrm{~N} / \mathrm{m}$ (MSNL, Veeco Instruments, Plainview, NY) were used for the indentation experiments. The exact spring constant of each cantilever was determined from the thermal noise spectrum (48-50). An example of a force-indentation curve recorded from the hydrogel is shown in (Figure S10). Elasticity fits (Hertz models) with conical tip geometry, corrected sample-tip distance and a Poisson ratio of 0.5 . were performed using JPK software. 


\section{Superresolution microscopy for bead position combined with Fura-2 Calcium imaging}

To obtain bead locations in 3D, we used TIRF microscopy. Measurements were performed using a custom-built single-molecule microscope. A 488-nm laser (200 mW; Coherent) was coupled through a single-mode fiber (QiOptics) into a Polytrope and Yanus (TILL Photonics) mounted on an inverted Zeiss Axiovert 200 microscope. The beam was then focused onto the back-focal plane of a high numerical aperture objective ( $\alpha-P l a n-A p o c h r o m a t ~ 100 \times / 1.46$; Zeiss) for highly inclined illumination (illumination intensity $\sim 1 \mathrm{~kW} / \mathrm{cm} 2$ ) or TIRF microscopy. Emission light was filtered using appropriate filter sets for Alexa 488. The emission light was imaged with a back-illuminated iXon DU 897 EMCCD camera (Andor Technology Ltd.), which was water-cooled to $-80{ }^{\circ} \mathrm{C}$. For functional calcium imaging the excitation was switched between 488nm and 340nm/380nm white light Halogen lamp, using the Polytrope (Till Photonics).

This allowed us to subsequently measure calcium and bead position every $500 \mathrm{~ms}$.

We estimated the lateral drift to be smaller than $50 \mathrm{~nm} / \mathrm{h}$, while drift correction was implemented at the level of the analysis software (see below). We observed no change in the widths of individual beads not exposed to cells during experiments, hence z-drift was not corrected.

\section{Localization Analysis.}

The data were analyzed as previously described (51). Briefly, smoothing, non-maximum suppression, and thresholding revealed the possible locations of fluorescent beads. Selected regions of interest were fitted by a pixelated Gaussian function and a homogeneous photon background with a maximum likelihood estimator for Poisson distributed data using a freely available fast GPU (graphics processing unit) fitting routine on a GeForce GTX1080 Ti (Nvidia).

We typically acquired 2000 frames for the reconstruction and data analysis of bead position. Lateral drift was corrected based on bead positions. The displacements corresponding to each time point were averaged using a robust estimator that was interpolated by a spline and used to correct the position of each localization. We estimated that the residual errors for the corrected positions were about $5 \mathrm{~nm}$.

The localization data were rendered using the Thomson blurring. Briefly, each localization is represented as a 2D Gaussian function with a width according to the precision of the respective localization determined from the fitted number of photons and background(52). All analysis software was written in MATLAB, based on the Single Molecule Analysis Platform (SMAP) by Ries lab (53). All quantitative analyses were done using the list of localization coordinates and not the processed images; the latter are only presented for visualization.

\section{Bead displacement to force calculations.}

To derive forces in normal and tangential direction we used the following formulas for spring coefficients (30)

$$
\begin{aligned}
& k_{\text {normal }} \approx \frac{\pi a E}{(1-v)(1+v)}=0.394 n N / \mu m, \\
& k_{\text {tangential }} \approx \frac{\pi a E}{(2-v)(1+v)}=0.14 n N / \mu m,
\end{aligned}
$$


where we estimate the Poisson ratio $v$ with 0.45 for our PEGDA hydrogel(27), the bead radius of $\mathrm{a}=100 \mathrm{~nm}$ and a Young's modulus $\mathrm{E}$ of $35 \mathrm{kPa}$ for the medium stiffness hydrogel in Figure 2. Forces in normal and tangential direction were calculated with:

$$
\begin{gathered}
F_{\text {normal }}=k_{\text {normal }} \Delta z \\
F_{\text {tangential }}=k_{\text {tangential }} \Delta \text { tang }
\end{gathered}
$$

where $\Delta$ tang $=\sqrt{\Delta x^{2}+\Delta y^{2}}$. Total forces were calculated from forces in normal and tangential direction.

$$
F_{\text {total }}=\sqrt{{F_{\text {normal }}^{2}+F_{\text {tangential }}^{2}}^{2}} \text {. }
$$

\section{Single Cell Calcium Imaging Analysis.}

All live-cell and fixed cell experiments were done with Jurkat T-cells (E6-1, ATCC), live-cell experiments were performed at $37^{\circ} \mathrm{C}$ adjusted by an objective heating system (OKO lab). Cells were loaded with $10 \mu \mathrm{M}$ Fura-2/AM (Molecular Probes ${ }^{\circledR}$ ) in complete RPMI 1640 at room temperature for $60 \mathrm{~min}$, washed with HBSS (Invitrogen) and flushed onto stimulatory surfaces on the stage of the above described custom built microscope. Cells were illuminated using a halogen lamp at 340/10nm and 380/10nm (polytrope, Till Photonics) and fluorescence emissions collected via a a-Plan-Apochromat 100x/1.46; Zeiss. Movies were acquired at a frame rate of 0.5 frames per second, the emission ratio of $340 / 380 \mathrm{~nm}$ was determined after background correction and preprocessed in Jupyter notebooks. Individual cells were selected manually and $\mathrm{Ca} 2+$ time traces were calculated after background correction. For noise reduction and elimination of outliers, a median filter with a window size of 10 time points was applied. The background for ratio of Fura-2 increase over time was calculated by averaging Fura-2 intensity from moment of cell touching down until Fura-2 increase.

\section{Immunostaining.}

Jurkat cells were seeded on the hydrogel surface $\left(50,000\right.$ cells per $\left.\mathrm{cm}^{2}\right)$ and incubated at $37^{\circ} \mathrm{C}$ and $5 \% \mathrm{CO}_{2}$ for $15-60 \mathrm{~min}$. The cells were then fixed in pre-warmed $4 \%$ PFA (Formaldehyde, Polysciences, Inc) and $0.1 \%$ Triton-X in Cytoskeletal buffer for $7 \mathrm{~min}$. The Cytoskeletal buffer consists of $10 \mathrm{mM}$ MES pH 6.1, $150 \mathrm{mM} \mathrm{NaCl}, 5 \mathrm{mM}$ EGTA, $5 \mathrm{mM}$ glucose and $5 \mathrm{mM} \mathrm{MgCl}_{2}$. For actin staining, the cells were pre-fixed with $0.5 \%$ PFA in Cytoskeletal buffer for $1 \mathrm{~min}$. The samples were then washed with PBS three times and incubated with $0.01 \% \mathrm{NH}_{4} \mathrm{Cl}$ in PBS for $10 \mathrm{~min}$ to reduce the autofluorescence. The samples were then washed with PBS for three times and were blocked with $2 \%$ BSA in PBS for $1 \mathrm{~h}$ at room temperature before staining. Phalloidin AlexaFluo 647 from Thermo Fisher Scientific in 1:200 dilution was used for actin staining. Anti-CD45 antibody (F10-89-4, Abcam and EP322Y, Abcam) and anti-CD3 antibody (clone SP7, ab16669, Abcam) in 1:200 dilutions were used to stain CD45 and TCR, respectively. The samples were then incubated with AlexaFluor 647-conjugated secondary 
antibodies for 1 hour at room temperature in the dark. The samples were then washed with PBS three times before mounting with Prolong gold antifade reagent (Molecular Probes).

\section{Airyscan Microscopy.}

For fixed cells imaging Zeiss LSM 880 Airyscan microscope was used to acquire images using 63x 1.4NA Oil DIC Plan-Apochromat objective. Argon $(488 \mathrm{~nm})$ and HeNe $(633 \mathrm{~nm})$ lasers were used to image the beads and the cells, respectively. Zen 2012 software was used as the interface for data acquisition.

Structured Illumination Microscopy. For super-resolution images, structured illumination microscopy (Zeiss Elyra) was used. Diode lasers with 488 and $561 \mathrm{~nm}$ were used for illumination, with a cyOFP specific $570-650 \mathrm{~nm}$ filter, where fluorescent beads FluoSpheres ${ }^{\mathrm{TM}}$ NeutrAvidin ${ }^{\mathrm{TM}}$-marked microspheres $(200 \mathrm{~nm}$ in diameter, fluorescent in yellow-green, ThermoFisher, F8774) were measured using a 495 - $550 \mathrm{~nm}$ emission filter. The signals were recorded by the Andor iXON 3885 camera. We used an alpha Plan-Apochromat 100× with 1.46 NA objective. The data was acquired with Zeiss Software, and was reconstructed and drift corrected with custom written Matlab code. We estimated the resolution of the microscope to be $160 \mathrm{~nm}$ in lateral and $350 \mathrm{~nm}$ axial direction for the actin structure, while bead position was estimated via wide field imaging and localization as described above. The cells were transfected with cyOFPtractin for $24 \mathrm{~h}$ prior cells seeding on hydrogels. Hydrogels were temperature equilibrated with culture media (RPMI) at $37^{\circ} \mathrm{C}$ Celsius for at least 1 hour prior cell seeding.

\section{Acknowledgement}

We thank Wes Legant and Michael L. Smith for general discussions and helpful advice regarding TFM and Daniel Nieves for advice with surface functionalization. We thank MZ Lin for kindly providing the cyOFP tractin plasmid. EK thanks for financial support from Human Frontiers Science Program RGY0065/2017, FEBS Long term fellowship throughout the onset of the project and ARC DECRA fellowship. MA funding for SNF Spark. GJS and JBH acknowledges funding from the Vienna Science and Technology Fund (WWTF) project LS13-030. 


\section{References}

1. J. F. Allard, O. Dushek, D. Coombs, P. A. van der Merwe, Mechanical modulation of receptor-ligand interactions at cell-cell interfaces. Biophys. J. 102, 1265-1273 (2012).

2. J. B. Huppa, M. M. Davis, T-cell-antigen recognition and the immunological synapse. Nat. Rev. Immunol. 3, 973-983 (2003).

3. F. Baumgart, M. Schneider, G. J. Schütz, How T Cells Do the "Search for the Needle in the Haystack." Frontiers in Physics 7 (2019).

4. E. Cai, et al., Visualizing dynamic microvillar search and stabilization during ligand detection by T cells. Science 356 (2017).

5. P. T. Sage, et al., Antigen recognition is facilitated by invadosome-like protrusions formed by memory/effector T cells. J. Immunol. 188, 3686-3699 (2012).

6. R. A. Fernandes, et al., A cell topography-based mechanism for ligand discrimination by the T cell receptor. Proceedings of the National Academy of Sciences 116, 14002-14010 (2019).

7. M. Lever, P. K. Maini, P. A. van der Merwe, O. Dushek, Phenotypic models of T cell activation. Nat. Rev. Immunol. 14, 619-629 (2014).

8. K. T. Bashour, et al., CD28 and CD3 have complementary roles in T-cell traction forces. Proc. Natl. Acad. Sci. U. S. A. 111, 2241-2246 (2014).

9. K. T. Bashour, et al., Cross Talk between CD3 and CD28 Is Spatially Modulated by Protein Lateral Mobility. Molecular and Cellular Biology 34, 955-964 (2014).

10. J. B. Huppa, et al., TCR-peptide-MHC interactions in situ show accelerated kinetics and increased affinity. Nature 463, 963-967 (2010).

11. M. Axmann, J. B. Huppa, M. M. Davis, G. J. Schütz, Determination of Interaction Kinetics between the T Cell Receptor and Peptide-Loaded MHC Class II via Single-Molecule Diffusion Measurements. Biophysical Journal 103, L17-L19 (2012).

12. J. Huang, et al., The kinetics of two-dimensional TCR and pMHC interactions determine T-cell responsiveness. Nature 464, 932-936 (2010).

13. V. Vogel, M. Sheetz, Local force and geometry sensing regulate cell functions. Nat. Rev. Mol. Cell Biol. 7, 265-275 (2006).

14. B. Liu, W. Chen, B. D. Evavold, C. Zhu, Accumulation of Dynamic Catch Bonds between TCR and Agonist Peptide-MHC Triggers T Cell Signaling. Cell 157, 357-368 (2014).

15. E. Klotzsch, G. J. Schütz, Improved Ligand Discrimination by Force-Induced Unbinding of the T Cell Receptor from Peptide-MHC. Biophysical Journal 104, 1670-1675 (2013).

16. W. Hwang, R. J. Mallis, M. J. Lang, E. L. Reinherz, The TCR mechanosensor exploits 
dynamic ectodomain allostery to optimize its ligand recognition site. Proc. Natl. Acad. Sci. U. S. A. 117, 21336-21345 (2020).

17. C. Zhu, W. Chen, J. Lou, W. Rittase, K. Li, Mechanosensing through immunoreceptors. Nat. Immunol. 20, 1269-1278 (2019).

18. S. T. Kim, et al., The alphabeta T cell receptor is an anisotropic mechanosensor. J. Biol. Chem. 284, 31028-31037 (2009).

19. Y. C. Li, et al., Cutting Edge: Mechanical Forces Acting on T Cells Immobilized via the TCR Complex Can Trigger TCR Signaling. The Journal of Immunology 184, 5959-5963 (2010).

20. J. Husson, K. Chemin, A. Bohineust, C. Hivroz, N. Henry, Force generation upon T cell receptor engagement. PLoS One 6, e19680 (2011).

21. Y. Feng, et al., Mechanosensing drives acuity of $\alpha \beta$ T-cell recognition. Proceedings of the National Academy of Sciences 114, E8204-E8213 (2017).

22. Y. Liu, et al., DNA-based nanoparticle tension sensors reveal that T-cell receptors transmit defined $\mathrm{pN}$ forces to their antigens for enhanced fidelity. Proc. Natl. Acad. Sci. U. S. A. 113, 5610-5615 (2016).

23. J. Göhring, et al., Temporal Analysis of T-Cell Receptor-Imposed Forces via Quantitative Single Molecule FRET Measurements. bioarxiv https:/doi.org/10.1101/2020.04.03.024299.

24. H. Colin-York, et al., Cytoskeletal Control of Antigen-Dependent T Cell Activation. Cell Reports 26, 3369-3379.e5 (2019).

25. Y. Jung, et al., Three-dimensional localization of T-cell receptors in relation to microvilli using a combination of superresolution microscopies. Proc. Natl. Acad. Sci. U. S. A. 113, E5916-E5924 (2016).

26. C. M. Kirschner, K. S. Anseth, Hydrogels in Healthcare: From Static to Dynamic Material Microenvironments. Acta Mater. 61, 931-944 (2013).

27. X.-H. Qin, et al., Enzymatic synthesis of hyaluronic acid vinyl esters for two-photon microfabrication of biocompatible and biodegradable hydrogel constructs. Polym. Chem. 5, 6523-6533 (2014).

28. W. R. Legant, et al., Measurement of mechanical tractions exerted by cells in three-dimensional matrices. Nat. Methods 7, 969-971 (2010).

29. U. S. Schwarz, et al., Calculation of forces at focal adhesions from elastic substrate data: the effect of localized force and the need for regularization. Biophys. J. 83, 1380-1394 (2002).

30. I. Schoen, B. L. Pruitt, V. Vogel, The Yin-Yang of Rigidity Sensing: How Forces and Mechanical Properties Regulate the Cellular Response to Materials. Annual Review of Materials Research 43, 589-618 (2013).

31. P. J. Diggle, A Point Process Modelling Approach to Raised Incidence of a Rare 
Phenomenon in the Vicinity of a Prespecified Point. Journal of the Royal Statistical Society. Series A (Statistics in Society) 153, 349 (1990).

32. S. V. Plotnikov, B. Sabass, U. S. Schwarz, C. M. Waterman, High-resolution traction force microscopy. Methods Cell Biol. 123, 367-394 (2014).

33. H. Cai, et al., Full control of ligand positioning reveals spatial thresholds for T cell receptor triggering. Nature Nanotechnology 13, 610-617 (2018).

34. A. M. Lipp, et al., Lck mediates signal transmission from CD59 to the TCR/CD3 pathway in Jurkat T cells. PLoS One 9, e85934 (2014).

35. E. Judokusumo, E. Tabdanov, S. Kumari, M. L. Dustin, L. C. Kam, Mechanosensing in T lymphocyte activation. Biophys. J. 102, L5-7 (2012).

36. A. Dey, A. K. Mukhopadhyay, Nanoindentation of Brittle Solids (CRC Press, 2014).

37. C. Wülfing, et al., Kinetics and extent of T cell activation as measured with the calcium signal. J. Exp. Med. 185, 1815-1825 (1997).

38. J. Chu, et al., A bright cyan-excitable orange fluorescent protein facilitates dual-emission microscopy and enhances bioluminescence imaging in vivo. Nature Biotechnology 34, 760-767 (2016).

39. E. Gutierrez, et al., High Refractive Index Silicone Gels for Simultaneous Total Internal Reflection Fluorescence and Traction Force Microscopy of Adherent Cells. PLoS ONE 6, e23807 (2011).

40. M. Fritzsche, et al., Self-organizing actin patterns shape membrane architecture but not cell mechanics. Nature Communications 8 (2017).

41. H. Kojima, A. Ishijima, T. Yanagida, Direct measurement of stiffness of single actin filaments with and without tropomyosin by in vitro nanomanipulation. Proc. Natl. Acad. Sci. U. S. A. 91, 12962-12966 (1994).

42. C. J. R. Sheppard, S. B. Mehta, R. Heintzmann, Superresolution by image scanning microscopy using pixel reassignment. Opt. Lett. 38, 2889-2892 (2013).

43. S. Halstenberg, A. Panitch, S. Rizzi, H. Hall, J. A. Hubbell, Biologically engineered protein-graft-poly(ethylene glycol) hydrogels: a cell adhesive and plasmin-degradable biosynthetic material for tissue repair. Biomacromolecules 3, 710-723 (2002).

44. C. N. Salinas, K. S. Anseth, Mixed Mode Thiol-Acrylate Photopolymerizations for the Synthesis of PEG-Peptide Hydrogels. Macromolecules 41, 6019-6026 (2008).

45. X. Z. Shu, et al., Attachment and spreading of fibroblasts on an RGD peptide-modified injectable hyaluronan hydrogel. J. Biomed. Mater. Res. A 68, 365-375 (2004).

46. B. Trappmann, et al., Extracellular-matrix tethering regulates stem-cell fate. Nat. Mater. 11, 642-649 (2012). 
47. O. Chaudhuri, et al., Substrate stress relaxation regulates cell spreading. Nat. Commun. $\mathbf{6}$, 6364 (2015).

48. R. W. Stark, T. Drobek, W. M. Heckl, Thermomechanical noise of a free v-shaped cantilever for atomic-force microscopy. Ultramicroscopy 86, 207-215 (2001).

49. J. L. Hutter, J. Bechhoefer, Calibration of atomic-force microscope tips. Review of Scientific Instruments 64, 1868-1873 (1993).

50. H.-J. Butt, M. Jaschke, Calculation of thermal noise in atomic force microscopy. Nanotechnology 6, 1-7 (1995).

51. E. Klotzsch, et al., Superresolution microscopy reveals spatial separation of UCP4 and F0F1-ATP synthase in neuronal mitochondria. Proceedings of the National Academy of Sciences 112, 130-135 (2015).

52. Y. Li, et al., Real-time 3D single-molecule localization using experimental point spread functions. Nat. Methods 15, 367-369 (2018).

53. J. Ries, SMAP: a modular super-resolution microscopy analysis platform for SMLM data. Nat. Methods (2020) https:/doi.org/10.1038/s41592-020-0938-1. 


\title{
Supplementary Information for
}

\section{Functionalized bead assay to measure 3-dimensional traction forces during T-cell activation}

\author{
Morteza Aramesh', Simon Mergenthal ${ }^{2}$, Marcel Issler², Birgit Plochberger ${ }^{3}$, Florian Weber ${ }^{3}$, \\ Xiao-Hua Qin ${ }^{4}$, Robert Liska ${ }^{5}$, Georg N. Duda ${ }^{6}$, Johannes B. Huppa ${ }^{7}$, Jonas Ries ${ }^{8}$, Gerhard J. \\ Schütz ${ }^{9,{ }^{*}}$ and Enrico Klotzsch ${ }^{1,2, *}$
}

${ }^{1}$ Laboratory of Applied Mechanobiology, Department for Health Sciences and Technology, ETH Zürich, 8093 Zürich, Switzerland

${ }^{2}$ Institute of Biology, Experimental Biophysics/ Mechanobiology, Humboldt Universität zu Berlin, 10115 Berlin, Germany

${ }^{3}$ Upper Austria University of Applied Sciences, Campus Linz, Garnisonstrasse 21, 4020 Linz, Austria

${ }^{4}$ Institute for Biomechanics, Department for Health Sciences and Technology, ETH Zürich, 8093 Zürich, Switzerland

${ }^{5}$ Institute of Applied Synthetic Chemistry, TU Wien, Getreidemarkt 9/163/MC, 1060, Vienna, Austria

${ }^{6}$ Julius Wolff Institute for Biomechanics and Musculoskeletal Regeneration, Charité-Universitätsmedizin Berlin

7 Institute for Hygiene and Applied Immunology, Center for Pathophysiology, Infectiology and Immunology, Medical University of Vienna, Vienna, Austria

${ }^{8}$ EMBL Heidelberg, Meyerhofstraße 1, 69117 Heidelberg, Germany.

${ }^{9}$ Institute of Applied Physics, TU Wien, Vienna, Austria

* Correspondence should be send to:

schuetz@iap.tuwien.ac.at and enrico.klotzsch@hu-berlin.de 
bioRxiv preprint doi: https://doi.org/10.1101/2020.09.23.310144; this version posted September 24, 2020. The copyright holder for this preprint (which was not certified by peer review) is the author/funder, who has granted bioRxiv a license to display the preprint in perpetuity. It is made available under aCC-BY-NC-ND 4.0 International license.

Correlation between normal and tangential forces

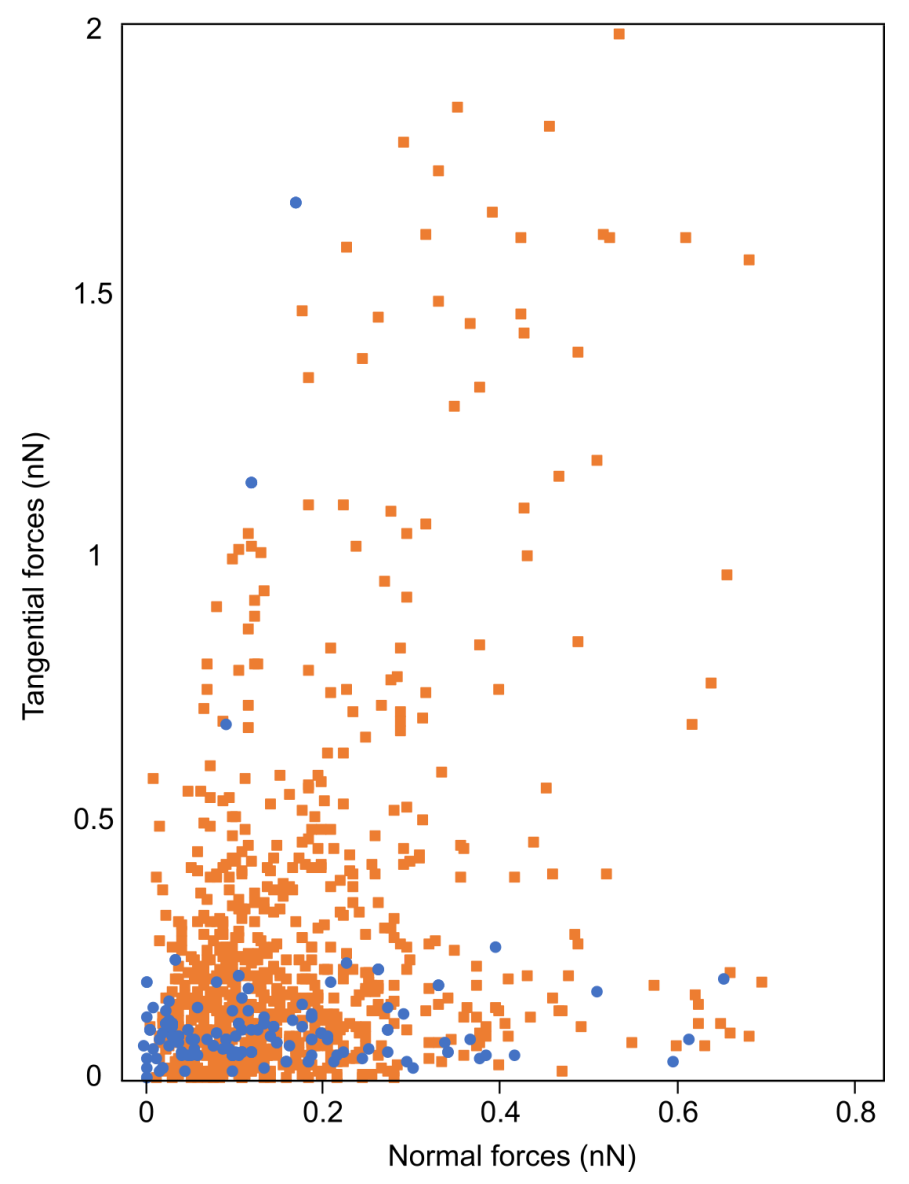

Figure S1: Correlation between normal and tangential forces. Data points from Figure $2 \mathrm{~d}$ are plotted as scatter plots, yielding a Spearman correlation of 0.09 for bead coated with anti-CD3 only (blue) and 0.25 for beads coated both with anti-CD3 and anti-CD28 (orange). 
Force - Calcium dependence
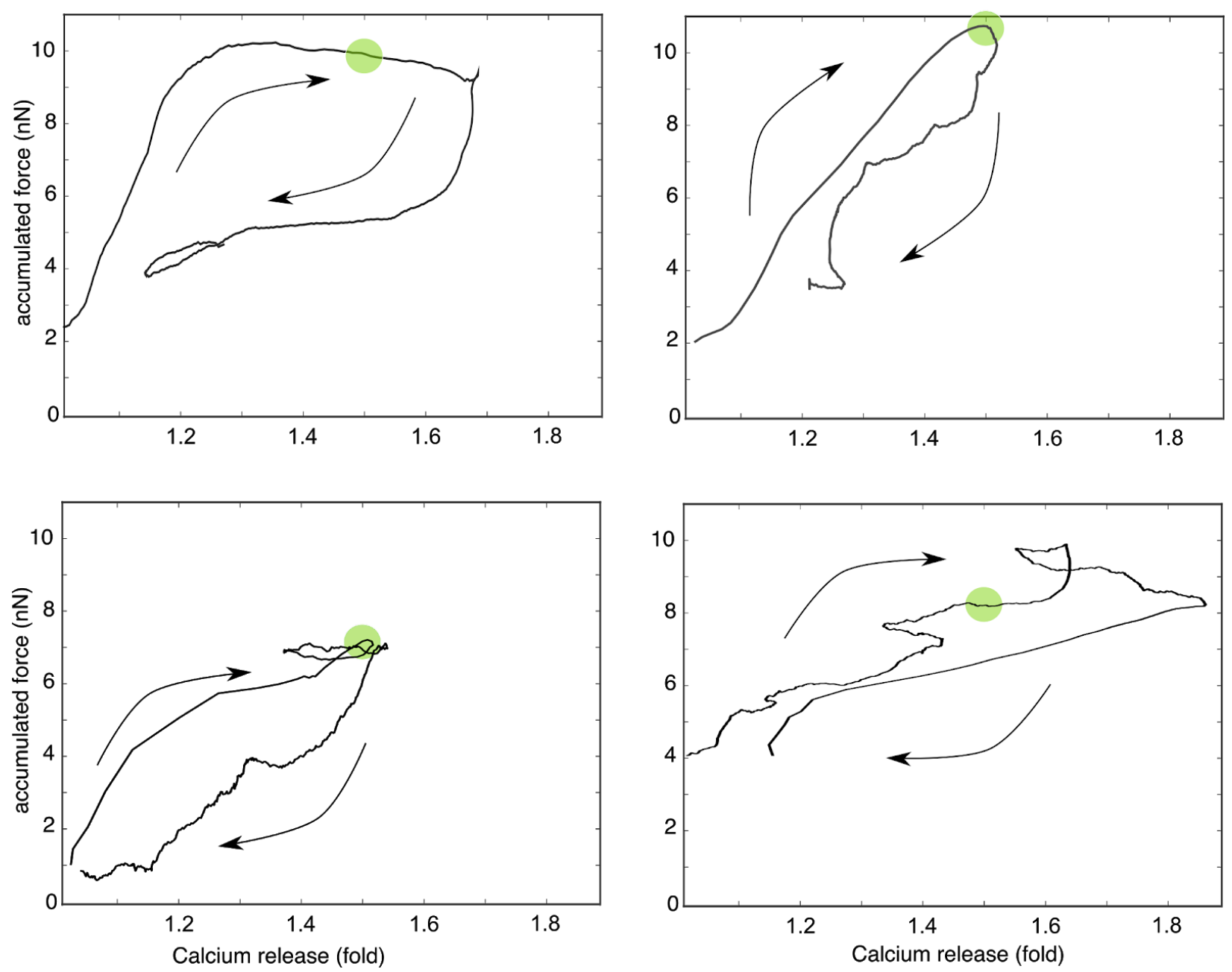

Figure S2: Four exemplary parametric force versus Fura-2 ratio plots are shown. T-cells were exposed to anti-CD3 and anti-CD28 coated beads. The measurements on the upper left corner represent data shown in Figure 2A-C. Green dot represents the time when $\mathrm{Ca}^{2+}$ ratio reached 1.5 fold increase. 
Time dependency of actin ring formation around the beads

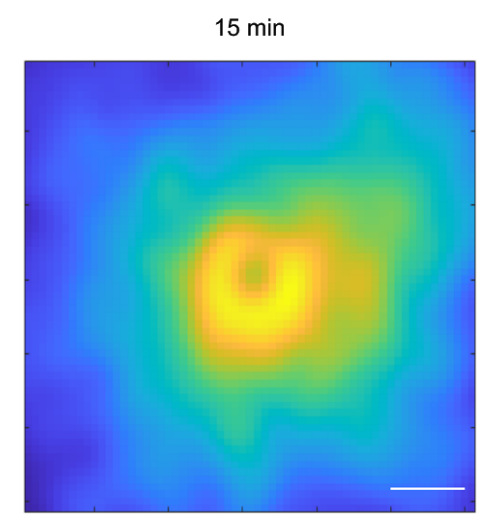

$30 \mathrm{~min}$
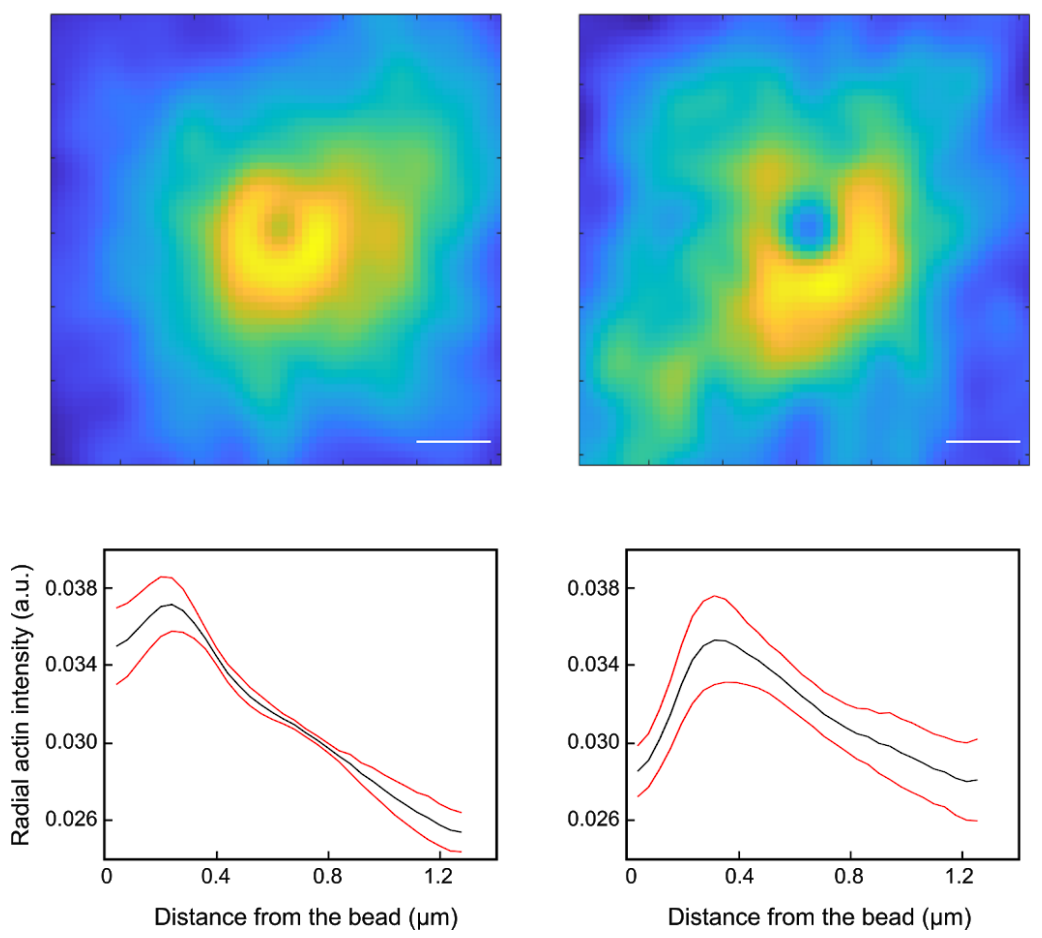
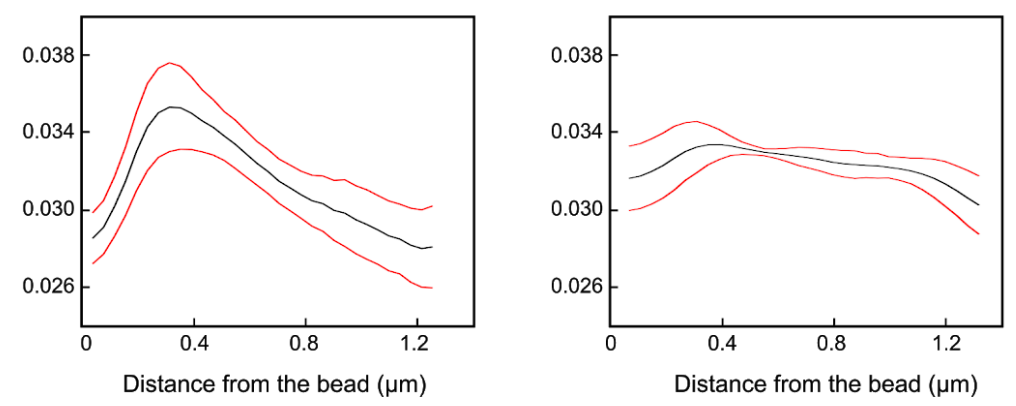

Figure S3: Additional experiments demonstrating actin ring formation. (a) The averaged actin intensity around activating beads for at least 10 cells is shown for $15 \mathrm{~min}, 30 \mathrm{~min}$ and $60 \mathrm{~min}$ after T-cell activation on anti-CD3/anti-CD28 coated beads. In contrast to Figure 3, cells were fixed before staining with phalloidin-A647. Scale bar is $400 \mathrm{~nm}$. (b) The average radial actin intensity is plotted as a black line for the same time points as in (a); red lines indicate with standard deviation derived from ten different cells with at least 30 beads. 


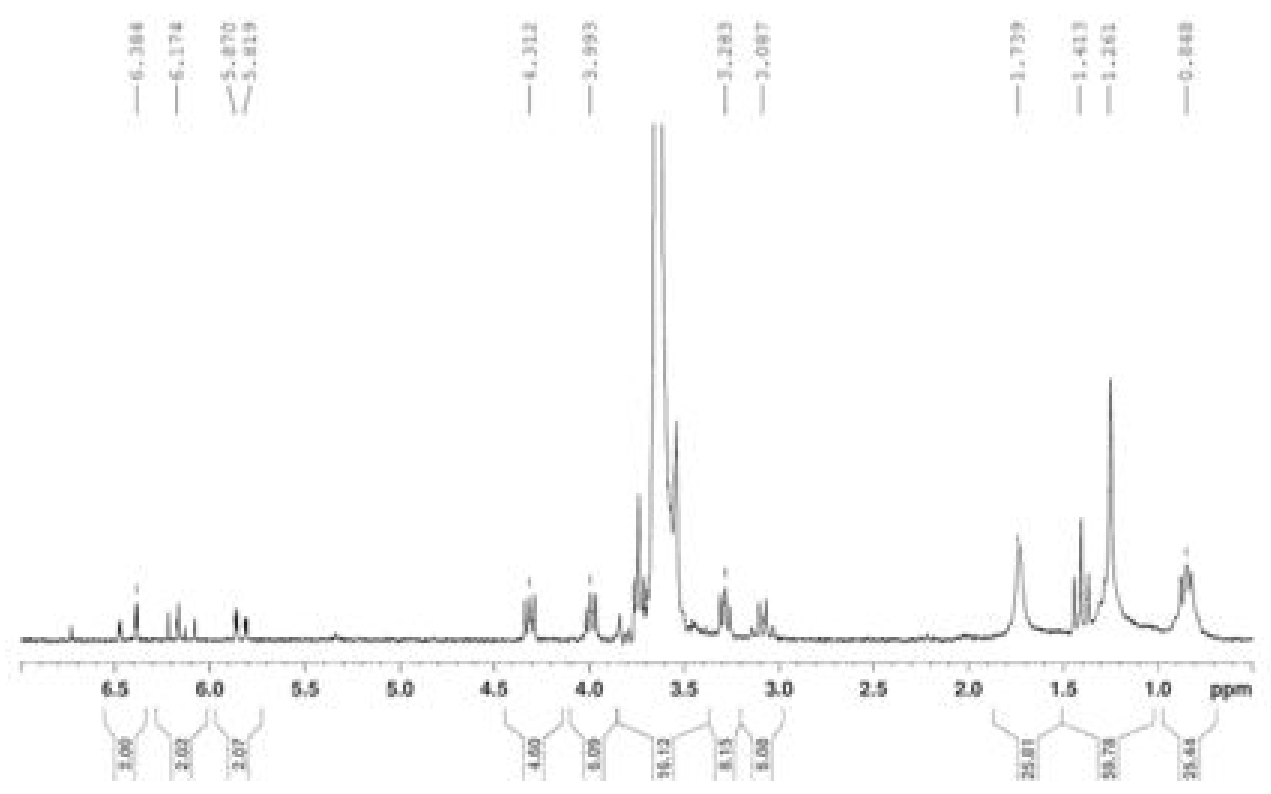

Figure S4. ${ }^{1} \mathrm{H}-\mathrm{NMR}$ spectrum of PEGDA-10k (200 MHz, $\left.\mathrm{CDCl}_{3}\right)$.

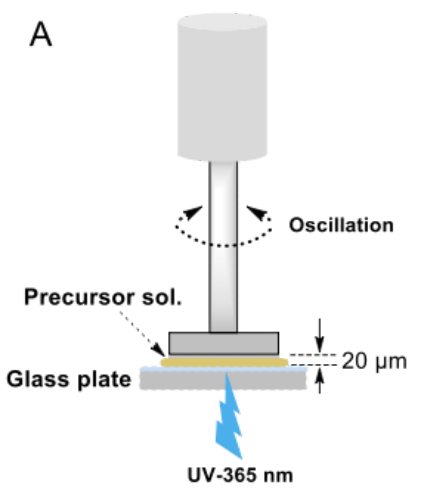

B

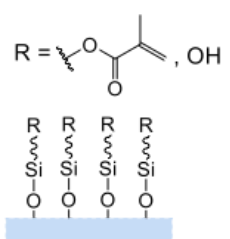

Figure S5. (A) Schematic showing the preparation of hydrogel thin layers on a photo-rheometer as well as the in situ oscillatory measurement of gel stiffness. (B) Schematic of the surface chemistry of methacrylated glass as the substrate for covalent fixation of PEG hydrogels. 


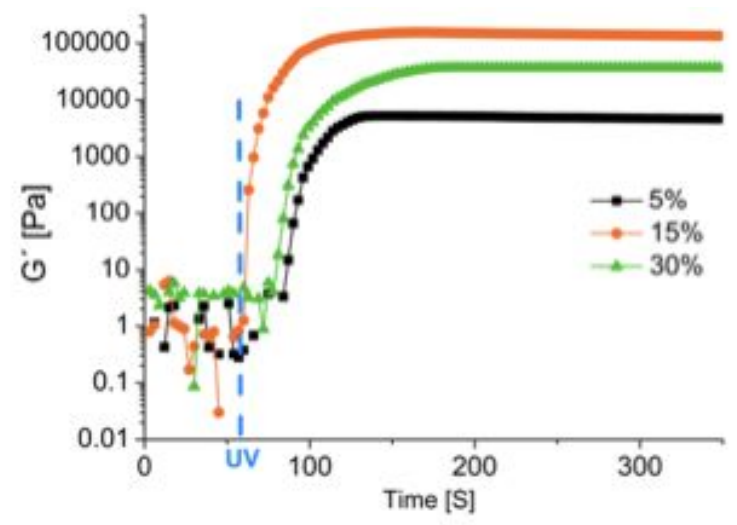

Figure S6: Photo-Rheology measurements for the different stiffness hydrogels. Young's modulus is plotted over the time of the UV crosslinking process. Saturation is reached after approximately 120 seconds at the target moduli defined by the chosen concentrations.
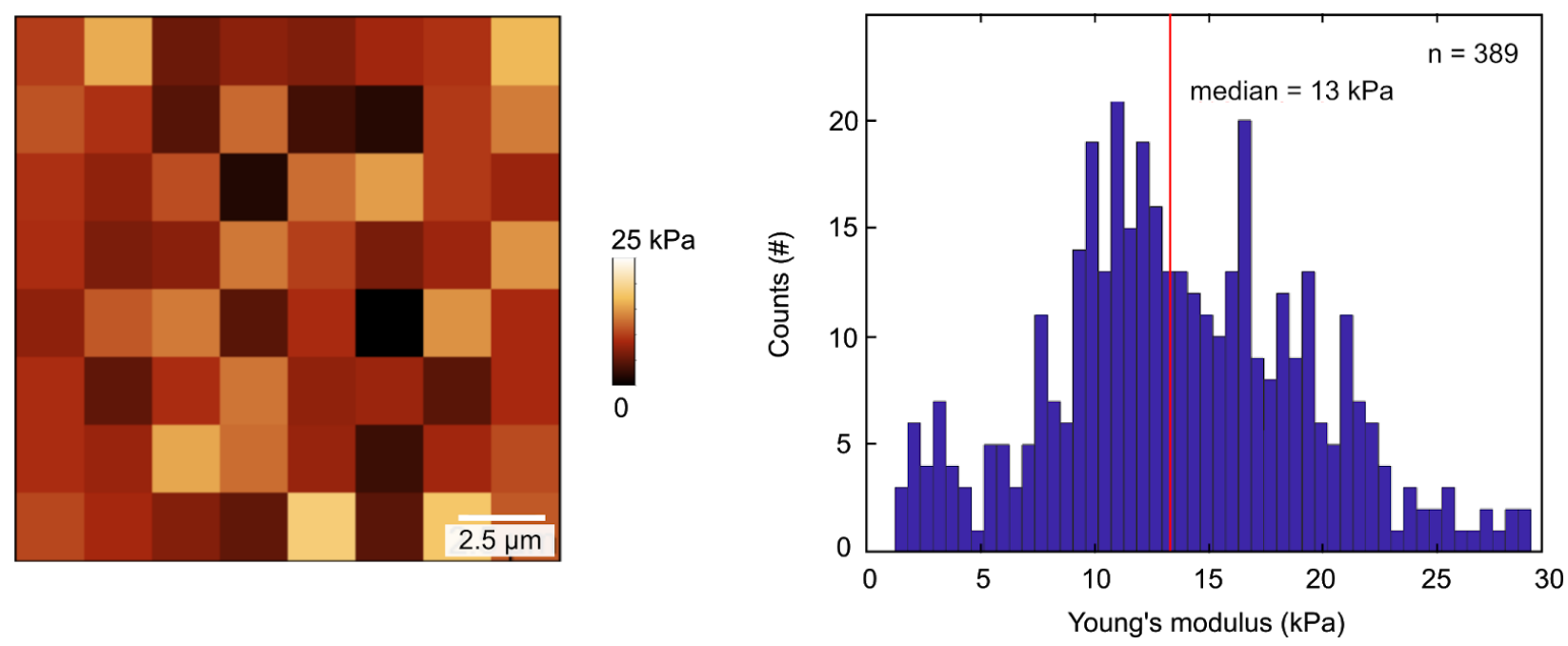

Figure S7: AFM indentation maps to measure Young's modulus of the Qgel920 based hydrogel. Young`s modulus maps are shown for mixing QGel components A and B (CHT, Quantum silicones) at a ratio of $1: 5$ arriving at a stiffness of $13.0 \pm 5.7 \mathrm{kPa}$ for the 20 by $20 \mu \mathrm{m}^{2}$ area. 
A

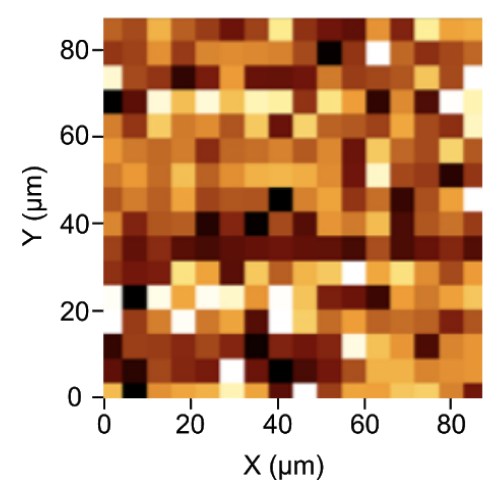

B

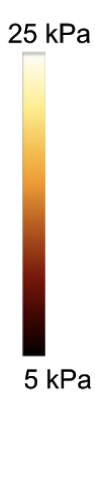

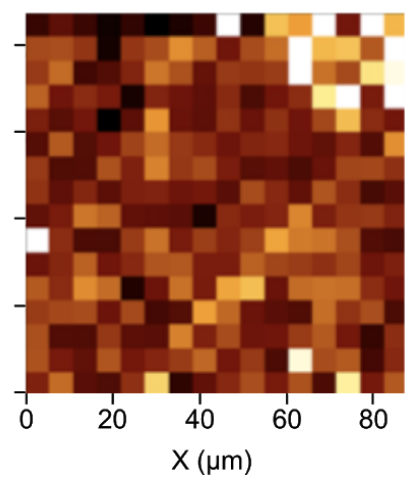

C

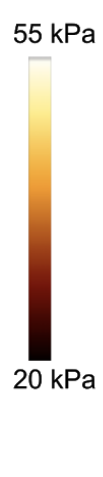

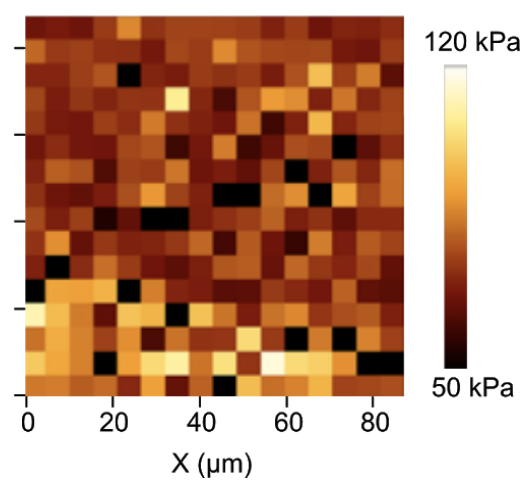

Figure S8: AFM indentation maps to measure Young's modulus of the hydrogel. Young's modulus maps are shown for (A) soft, (B) medium and (C) hard PEGDA gels; indentation measurements were performed every 5.6 micrometer. We calculated mean values of $14 \pm 5 \mathrm{kPa}(\mathbf{A}), 33 \pm 14 \mathrm{kPa}(\mathbf{B})$ and $95 \pm 19 \mathrm{kPa}(\mathbf{C})$.

Loading rate dependence of Young's modulus

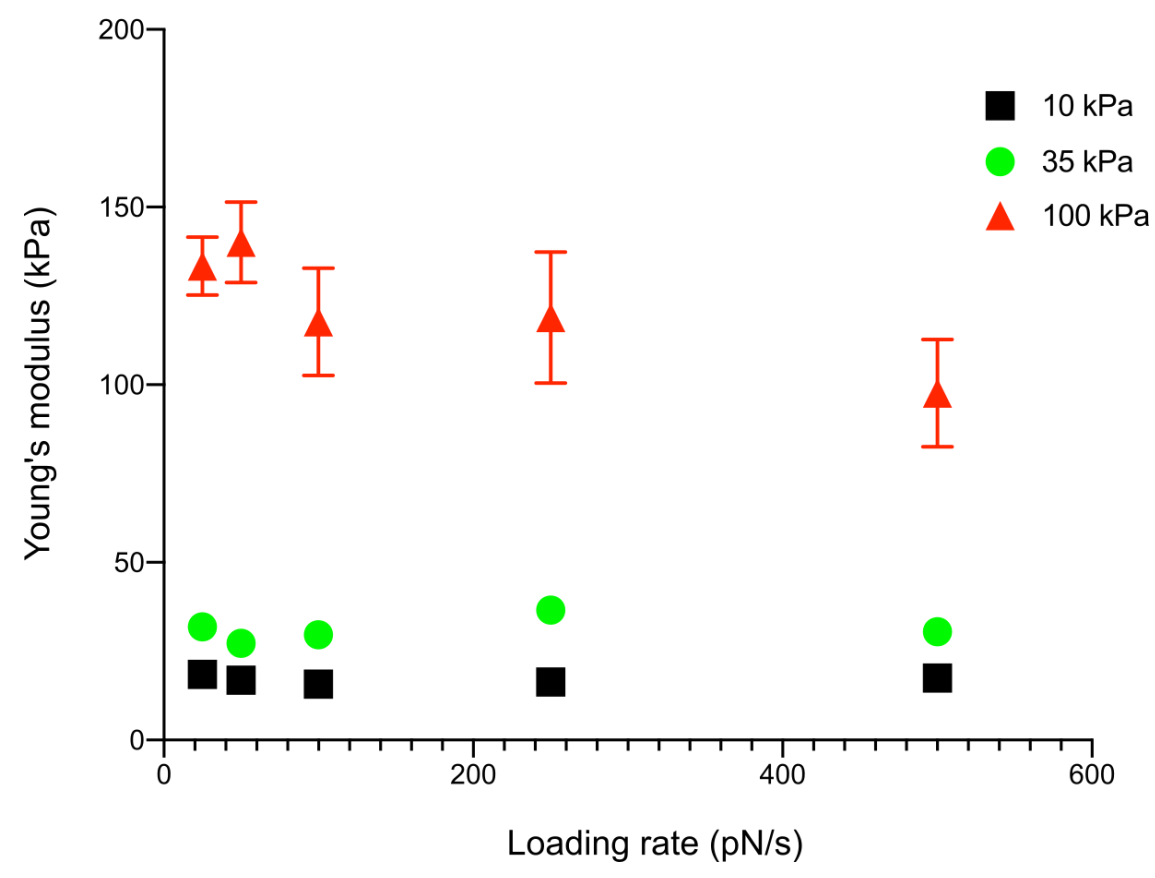

Figure S9: AFM indentation loading rate dependence of different maps to measure Young's modulus of the hydrogel. Young`s modulus maps are shown for soft, medium and hard PEGDA gels. 
bioRxiv preprint doi: https://doi.org/10.1101/2020.09.23.310144; this version posted September 24, 2020. The copyright holder for this preprint (which was not certified by peer review) is the author/funder, who has granted bioRxiv a license to display the preprint in perpetuity. It is made available under aCC-BY-NC-ND 4.0 International license.

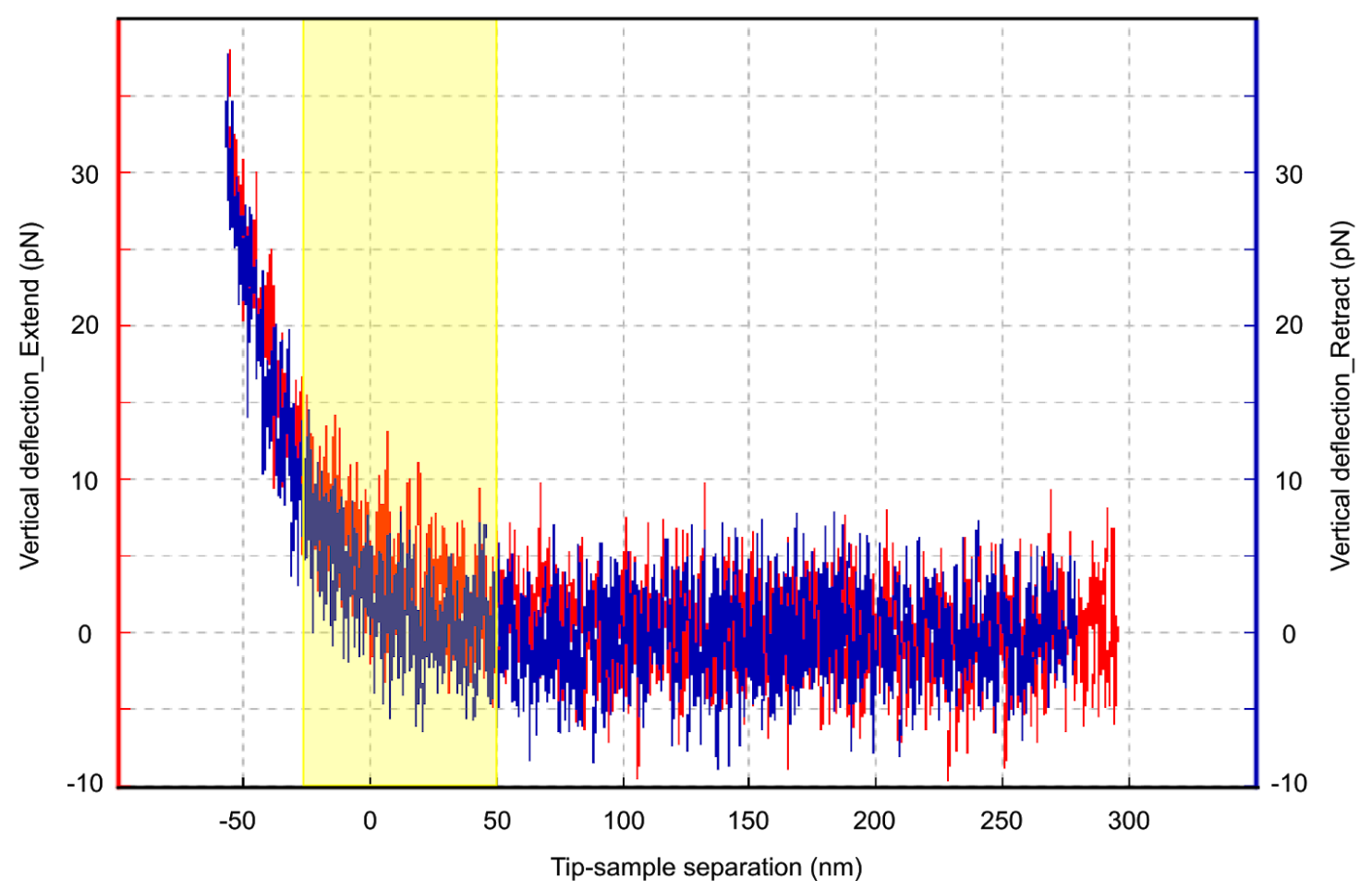

Figure S10: An example AFM tip indentation curve performed on a hydrogel is shown. Approach Curve (Red), Retrace (Blue), Fitted region (Hertz-Model) (Yellow). The measurement was performed using a cantilever with a spring constant of $10 \mathrm{pN} / \mathrm{nm}$, a tip velocity of $1 \mu \mathrm{m} / \mathrm{sec}$ and a set trigger force of $35 \mathrm{pN}$.

Movie S1: Actin dynamics during T-cell bead interaction visualized by SIM. Fluorosphere beads are in grey, actin is visualised with cyOFP-tractin in green. The total length of the movie represents 50 minutes in real time. 